\author{
混合导体透氧膜反应器中水分解反应研究进展 \\ 蔡莉莉 $a, b$ 王静忆 $a, b$ 朱雪峰*, $a, b, c$ 杨维慎 $a, b$ \\ ( ${ }^{a}$ 中国科学院大连化学物理研究所 催化基础国家重点实验室 大连 116023) \\ ( $b$ 中国科学院大学 北京 100049$)$ \\ ( ${ }^{c}$ 中国科学院洁净能源创新研究院 大连 116023)
}

\begin{abstract}
摘要 混合导体透氧膜反应器可以将供氧反应、氧分离和耗氧反应耦合在一个单元, 实现反应和分离一体化, 简化化 工过程. 水分解反应参与的混合导体透氧膜反应器能够实现氢气的制备和分离, 近年来受到越来越多的关注. 这篇文 章综述了混合导体透氧膜反应器中水分解反应领域的研究进展, 总结了包括膜材料、催化剂、操作条件等对透氧膜反 应器中水分解反应的影响, 分析了目前存在的问题, 同时展望了该领域在膜材料、膜结构和催化剂开发等方面的未来 发展方向, 希望有助于促进膜反应器中水分解反应的研究.
\end{abstract}

关键词 氢气; 透氧膜; 水分解; 膜材料; 催化剂

\title{
Recent Progress on Mixed Conducting Oxygen Transport Membrane Reactors for Water Splitting Reaction
}

\author{
Lili Cai $^{a, b} \quad$ Jingyi Wang ${ }^{a, b} \quad$ Xuefeng Zhu* ${ }^{*, a, b} \quad$ Weishen Yang ${ }^{a, b}$ \\ ( ${ }^{a}$ State Key Laboratory of Catalysis, Dalian Institute of Chemical Physics, Chinese Academy of Sciences, \\ Dalian 116023, China) \\ ( ${ }^{b}$ University of Chinese Academy of Sciences, Beijing 100049, China) \\ ( ${ }^{c}$ Dalian National Laboratory for Clean Energy, Chinese Academy of Sciences, Dalian 116023, China)
}

\begin{abstract}
Catalytic membrane reactors based on mixed conducting oxygen transport membranes (OTMs) have the ability to integrate reaction and separation, and thus simplify the chemical process by coupling oxygen supplying reaction, oxygen separation and oxygen consumption reaction into a single unit. In recent years, more and more attentions have been paid to the mixed conducting OTM reactors for water splitting reaction owing to their applications for preparation and separation of hydrogen. In this article, we summarize the recent progress on mixed conducting OTM reactors for water splitting reaction, including the effects of membrane materials, catalysts and operation conditions on the water splitting involved membrane reactors. Meanwhile, the current existing problems in the above aspects are analyzed, and the future development of membrane materials, membrane structures and catalysts in this field is prospected. We wish this paper will be helpful for the development of the water splitting in membrane reactors.

Keywords hydrogen; oxygen transport membrane; water splitting; membrane material; catalyst
\end{abstract}

\section{1 引言}

混合导体透氧膜 (OTM) 是一类在高温 (尤其是 $700{ }^{\circ} \mathrm{C}$ 以上) 同时具有氧离子和电子导电性能的致密陶 瓷膜. 在氧气分离过程中, 氧气以氧离子的形式通过混 合导体 OTM 中的晶格氧或者间隙氧进行传递, OTM 对 氧气有 $100 \%$ 的渗透选择性. 基于混合导体 OTM 构建的 催化膜反应器可以耦合供氧反应、氧分离和耗氧反应, 实现反应和分离一体化, 简化化工过程. OTM 反应器的 研究早已不局限于简单的氧分离制取高纯氧 $[1-10]$, 以及 耦合低碳烷烃或焦炉煤气选择性氧化反应等 ${ }^{[11-20]}$. 近年
来, 研究者们通过改变供氧和需氧反应, 发展了多种新

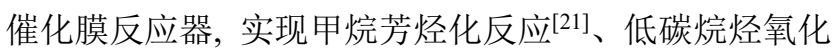

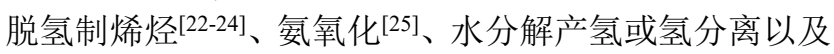
同时得到液体合成气和氨合成气等 ${ }^{[26-36]}$.

为了应对全球变暖等气候变化, 低碳减排已成为全 球范围内的大趋势，世界能源结构从传统化石能源为主 转向以可再生能源为主的多元格局. 氢气作为环境友好 的可再生能源载体，在化工、航天、燃料电池等领域的 需求越来越大, 它的制备和分离是氢能源体系中的重要 环节. 水分解制氢是一种环境友好的方法, 根据不同的 分解方式可以分为电解水、光解水和热解水制氢. 电解

* E-mail: zhuxf@dicp.ac.cn

Received December 9, 2020; published January 25, 2021.

Project supported by the National Natural Science Foundation of China (Nos. 22008231, 21776267), Dalian National Laboratory for Clean Energy (DNL) (DNL180203), and LiaoNing Revitalization Talents Program (XLYC1801004).

项目受国家自然科学基金(Nos. 22008231, 21776267)、中国科学院洁净能源创新研究院(DNL180203)和辽宁省 “兴辽英才” 计划(XLYC1801004)资助. 
水制氢能得到高纯氢气，技术相对成熟，已经实现工业 化应用. 但电解槽的高耗电量使其能量转化效率长期徘 徊在 50\% 70\%之间, 是电解水生产成本居高不下的主 要原因之一, 无法与其他大型制氢技术相竞争 ${ }^{[37-38]}$. 光 解水制氢是使用一些光催化剂吸收可见光的能量, 然后 将水分解成 $\mathrm{H}_{2}$ 和 $\mathrm{O}_{2}$. 由于缺乏有效的光催化剂, 光解 水制氢的产氢速率较低, 是目前能量转化效率最低的过 程, 仅有 $0.06 \%$, 相应的产氢成本较高, 距离商业化应 用的要求还是遥遥无期 ${ }^{[37-40]}$. 热解水制氢是一种更有效 的产氢方式, 其能量转化效率接近 $50 \%$, 但受限于氢氧 分离以及分解温度高的问题. 目前研究者们已经通过热 化学循环和催化膜反应器等方式将水分解温度降至合 适温度 ${ }^{[37-41]}$. 依赖于这些技术的突破, 热解水制氢将成 为最有希望实现工业化规模应用的技术方式. 近年来, 利用混合导体 OTM 反应器可以实现原位移除水分解反 应中的氧, 得到高纯氢气. 因其可謧美 Pd膜的产氢速率 及理论上 $100 \%$ 的氢气选择性, 相关的研究得到越来越 多的关注. 本综述总结了近年来该类膜反应器的研究进 展, 主要涉及膜材料、膜结构、催化剂以及影响膜反应 器性能的因素等方面.

\section{2 混合导体 OTM 反应器中的水分解反应}

从概念上来讲, 水分解成氧和氢的反应很简单, 但 实现起来却很困难. 因为它是一个高度吸热的热力学受 限反应. 大量的模拟研究和实验结果表明, 超过 $2200{ }^{\circ} \mathrm{C}$ 才能达到一定程度的水分解 ${ }^{[42-46]}$. 即使在 $1600{ }^{\circ} \mathrm{C}$, 水分解产生的氢气浓度也仅有 $0.1 \%$. 利用混 合导体 OTM 反应器耦合水分解反应, 可以极大程度地 提高水分解反应的转化率. 具有不同氧移除反应的水分 解催化膜反应器的示意图如图 1 所示. 1995 年, Naito 和 Arashi ${ }^{[47]}$ 首次使用 $\mathrm{TiO}_{2}$ 掺杂的 YSZ膜材料, 通过调控膜 反应器另一侧的氧分压, 在 $1600{ }^{\circ} \mathrm{C}$ 实现水分解反应, 产生的氢气浓度达到 $1 \% \sim 11 \%$. 但受限于膜材料的电 子电导率, 在 $1600{ }^{\circ} \mathrm{C}$ 其水分解速率仅约 $0.5 \mathrm{~mL} \cdot \mathrm{cm}^{-2}$ • $\mathrm{min}^{-1}$. 超高的操作温度导致密封困难和高能耗, 阻碍了 其应用. 后来, Balachandran 等 ${ }^{[48]}$ 通过在 $\mathrm{Gd}$ 掺杂的 $\mathrm{CeO}_{2}$ 氧化物(GDC)中加入 $40 \%$ (体积比)的金属 $\mathrm{Ni}(\mathrm{Ni}-\mathrm{GDC})$ 来 增加膜材料的电子电导率, 将膜反应器的操作温度降至 $900{ }^{\circ} \mathrm{C}$, 水分解速率达到约 $6.0 \mathrm{~mL} \cdot \mathrm{cm}^{-2} \cdot \mathrm{min}^{-1}$. 该膜反 应器中, 耦合了水分解反应和氢氧化反应, 增加了水分 解反应的转化率. 但同时他们也指出这种做法仅作为评 价膜反应器产氢速率的一种方法, 没有实际意义. 随后, Jiang 等 ${ }^{[28]}$ 在混合导体 OTM 反应器中耦合水分解反应和 甲烷部分氧化反应(POM), 同时得到高纯氢气和合成 气. 近年来, $\mathrm{Li}$ 和 Cai 等 ${ }^{[27,49-53]}$ 提出在混合导体 OTM 反 应器中耦合水分解反应和氢氧化反应, 能实现氢分离. 虽然在该 OTM 反应器中, 净反应为零, 但膜两侧发生 了物质的交换, 实现了低纯氢制高纯氢. 在后续的研究
中, 他们优化多种膜材料和催化剂, 使得水分解转化率 高达 $42 \%{ }^{[52]}$. 在膜的另一侧引入还原性气体如甲烷、 $\mathrm{CO}$ 或 $\mathrm{H}_{2}$ 等均能提高水侧的水分解转化率，促进该类膜 反应器在水分解产氢或氢分离方面的应用.

混合导体 OTM 的氧渗透机理已经在多个文献中详 细介绍, 这里不再赘述. 水分解反应参与的混合导体 OTM 反应器的基本原理如下, 膜一侧的供氧源是水(水 侧), 另一侧的耗氧源是还原性气体, 如低碳烷烃、 CO 或 $\mathrm{H}_{2}$ 等(图 1). 在高温下, 水发生热分解反应, 生成氢气 和氧离子, 氧离子在氧化学势梯度的驱动下通过 OTM 体相传输到耗氧反应侧并与另一侧的还原性气体反应. 由于混合导体 OTM 只能传导氧离子, 所以水分解产生 的氧离子可被选择性移除, 促进了水分解反应平衡向右 移动, 增加了水分解反应的转化率, 也即提高氢生成速 率. 水侧的出口气体经过冷凝去除未反应完的水, 即可 得到高纯氢气. 当耗氧侧的还原气体为低纯 $\mathrm{H}_{2}$ 时, 低纯 $\mathrm{H}_{2}$ 和渗透过来的氧离子反应生成水和电子. 电子再通 过膜体相传输到水侧, 在膜体相保持电荷平衡. 水侧产 生的氢气量等于另一侧消耗的氢气量. 简言之, 在上述 过程中没有发生净反应，但是在膜两侧实现了物质交 换. 整个过程消耗了低纯度氢气, 获得了高纯度氢气. 所以, 这个过程可以认为是一个氢分离的过程, 即利用 反应-反应耦合实现了氢分离. 耦合水分解反应的混合 导体 OTM 反应器的氢气生成速率、氢分离速率和水分 解速率相等, 均是膜透氧量的 2 倍.

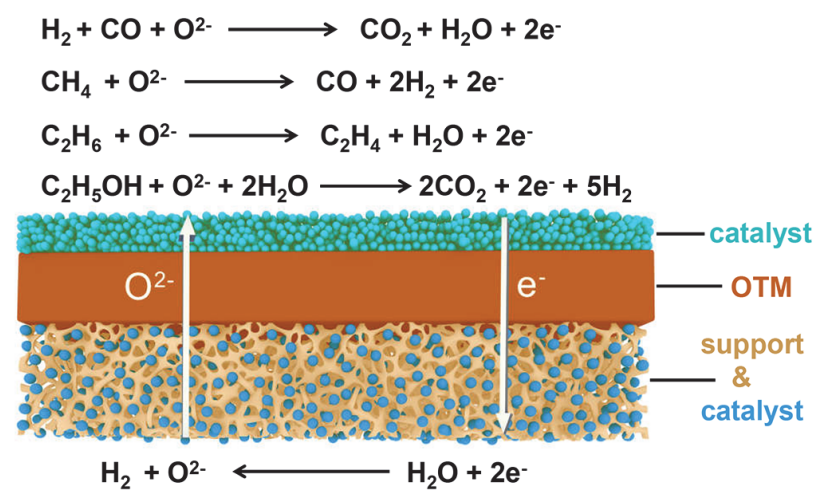

图 1 不同氧移除反应的水分解催化膜反应器

Figure 1 The catalytic membrane reactors for water splitting with different oxygen removal reactions

这类膜反应器主要涉及三个过程, 分别是发生在水 侧催化剂层和膜表面的水分解反应、膜体相的氧扩散过 程，以及发生在催化剂层和膜表面的还原性气体被氧化 的过程. 膜材料、催化剂和膜两侧的氧分压梯度等都会 影响膜反应器的性能. 这种催化膜反应器中, 膜两侧均 处于还原性气氛中, 对膜材料和催化剂的稳定性要求也 更高. 由于膜两侧所处的氧分压不同于单纯的氧分离过 程, 材料的电导率和稳定性也不同. 所以, 设计满足需 求的膜材料和催化剂在耦合水分解反应的混合导体 OTM 反应器中有重要意义. 在本综述中, 有关催化剂 
的设计方面重点关注水分解反应侧的催化剂.

\section{3 膜材料}

在混合导体 OTM 反应器中进行水分解反应可以极 大地提高水分解反应的转化率, 膜材料和催化剂对膜反 应器的性能起决定性作用. 在该类膜反应器中, 膜两侧 均处在还原性气氛中, 对材料的稳定性要求高. 在空气 分离制纯氧应用中具有良好稳定性的膜材料, 不一定适 用于水分解反应. 发展同时具有稳定性好和透氧量高的 材料是 OTM 领域极具挑战性的课题. 下面将详细介绍 膜材料在水分解反应中的应用以及其他影响膜反应器 性能的因素.

\section{1 混合导体 OTM 材料简介}

经过几十年的发展, 研究者们已经笁选出了一些具 有应用前景的 OTM 材料. 混合导体 OTM 的氧渗透性 能、化学稳定性、机械强度、热膨胀性、高温密封和材 料成本都将决定其在实际体系中的应用. 目前萤石型和 钙钛矿型是研究及应用较广泛的材料. 萤石型材料主要 有 $\mathrm{ZrO}_{2}$ 型、 $\mathrm{CeO}_{2}$ 型和 $\delta-\mathrm{Bi}_{2} \mathrm{O}_{3}$ 型等. 钙钛矿型(其一般 式为 $\mathrm{ABO}_{3}$ )材料是目前研究最多, 且透氧量最高的材 料. 由于钻钛矿结构的离子掺杂可变性非常大, 元素周 期表中 $90 \%$ 的金属元素都可以形成钙钛矿结构. 因此, 钲钛矿型材料也是被研究最广泛、最深入的一类 OTM 材料. 多篇综述及专著中已经详细介绍了这些材料的基 本信息 ${ }^{[8,14,54-58]}$.

根据材料的物相组成可以将 OTM 分为单相膜、双 相膜或多相膜. 单相混合导体 OTM, 顾名思义就是由 单一物相组成的膜. 单相膜组成简单, 透氧量较高, 一 直是 OTM 领域的研究重点. 很多含 Co 单相膜的氧渗透 性较高, 但在还原性气氛中稳定性差, 严重制约了它 们用作膜反应器. 为了解决膜材料在还原气氛下稳定性 差的问题, 研究者们开发了双相混合导体 OTM. 双相 混合导体 OTM 材料中存在两种不同的物相. 按照两相 组成的不同, 双相膜可以分为氧离子导体-纯电子导体 双相膜、氧离子导体-混合导体双相膜和混合导体-混合

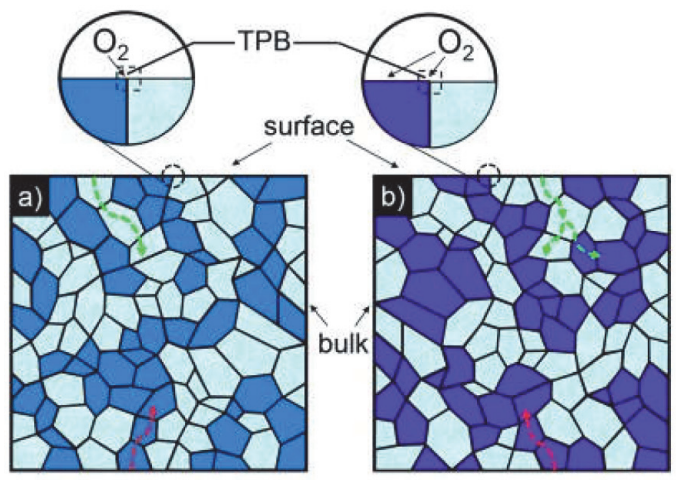

导体双相膜三大类 ${ }^{[59-75]}$, 如图 2 所示. 氧离子导体-纯电 子导体双相膜中氧离子通过氧离子导体传输，电子通过 纯电子导体反向传输. 氧离子导体-混合导体双相膜中 氧离子通过氧离子导体和混合导体传输, 电子通过混合 导体反向传输. 混合导体-混合导体双相膜中氧离子和 电子可同时通过这两相传输. 双相膜的两相之间要有良 好的化学相容性, 即不能发生化学反应. 下面将详细介 绍这两类材料在水分解反应中的应用.

\section{1 .1 单相 OTM 材料}

单相 OTM 材料的组成简单, 且透氧量较高, 有利 于移除水分解产生的氧离子. 研究者们考察了不同单相 膜材料在参与水分解反应时的水分解性能，总结在表 1 中. Evdou等[76]考察了 $\mathrm{La}_{0.3} \mathrm{Sr}_{0.7} \mathrm{FeO}_{3-\delta}$ 管状膜反应器在使 用不同的氧移除模式时对水分解速率的影响, 如使用 惰性气体 $\mathrm{He}$ 吹扫移除氧时, 该膜反应器在 $860{ }^{\circ} \mathrm{C}$ 的水 分解速率仅约 $0.005 \mathrm{~mL} \cdot \mathrm{cm}^{-2} \cdot \mathrm{min}^{-1}$. 当使用 $\mathrm{CO}$ 作为还 原性气体来移除氧时, 相同条件下的水分解速率增加了 两倍. 这直接证明了增大氧分压梯度有利于氧的移除, 进而增加了水分解速率. Jiang 等 ${ }^{[28-29,77]}$ 在 $\mathrm{BaZr}_{x} \mathrm{Co}_{y^{-}}$ $\mathrm{Fe}_{z} \mathrm{O}_{3-\delta}(x+y+z=1)(\mathrm{BZCF})$ 中空纤维膜反应器中用惰性 气体 $\mathrm{He}$ 来原位移除水分解产生的氧, 在 $900{ }^{\circ} \mathrm{C}$ 的水分 解速率非常低 $\left(<0.026 \mathrm{~mL} \cdot \mathrm{cm}^{-2} \cdot \mathrm{min}^{-1}\right)$. 当耦合 $\mathrm{POM}$ 反 应来原位移除水分解产生的氧时, 在 $900{ }^{\circ} \mathrm{C}$ 的水分解 速率达到 $2.2 \mathrm{~mL} \cdot \mathrm{cm}^{-2} \cdot \mathrm{min}^{-1}$, 同时 POM 侧还能得到合 成气[28-29,77]. $\mathrm{Li}$ 等 ${ }^{[49]}$ 在 $0.5 \mathrm{~mm}$ 厚的 $\mathrm{Ba}_{0.98} \mathrm{Ce}_{0.05} \mathrm{Fe}_{0.95} \mathrm{O}_{3-\delta}$ (BCF) 膜反应器中, 分别使用合成气和不同纯度的氢气 来原位移除水分解产生的氧, 结果表明使用氢气作为还 原性气体时能得到更高的水分解速率. 在氢气纯度为 $80 \%$ 时，该膜反应器在 $900{ }^{\circ} \mathrm{C}$ 的水分解速率达到 10.2 $\mathrm{mL} \cdot \mathrm{cm}^{-2} \cdot \mathrm{min}^{-1}$. 作者认为在对氧移除反应上 $\mathrm{CO}$ 比 $\mathrm{H}_{2}$ 的 反应活性低，同时产生的 $\mathrm{CO}_{2}$ 部分占据了催化剂的活性 位点，导致氢氧化反应的活性位点数目减少. 这两方面 的原因使得合成气作为还原气体移除氧离子时的水分 解速率较使用氢气时低. 虽然在 $\mathrm{BCF}$ 膜材料上能得到 较高的水分解速率, 但在 $100 \mathrm{~h}$ 的连续稳定性测试中,

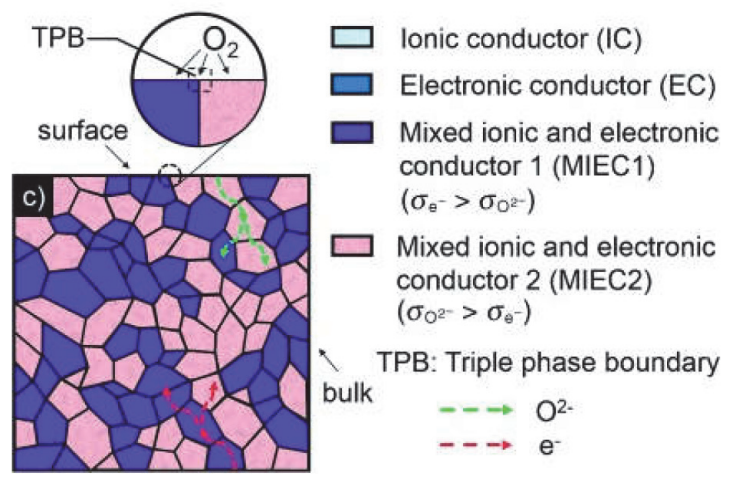

图 2 双相膜示意图 ${ }^{[75]}$. (a)氧离子导体-纯电子导体, (b)氧离子导体-混合导体, (c)混合导体 1-混合导体 2 双相膜

Figure 2 Schematic diagrams of dual-phase membranes ${ }^{[75]}$. (a) IC-EC (ionic conductor-electronic conductor), (b) IC-MIEC1 (mixed ionic and electronic conductor), (c) MIEC1-MIEC2. Reprinted with permission from ref. [75], (C) 2015 John Wiley and Sons 
表 1 单相透氧膜材料的水分解性能

Table 1 The water splitting performance of single-phase oxygen transport membranes

\begin{tabular}{|c|c|c|c|c|c|c|}
\hline Membrane materials & Thickness/mm & $T /{ }^{\circ} \mathrm{C}$ & $\begin{array}{l}\text { Atmospheres } \\
\text { at both sides }\end{array}$ & $\begin{array}{c}\text { Catalysts } \\
\text { at water side }\end{array}$ & $\begin{array}{c}F_{\mathrm{H}_{2}} / \\
\left(\mathrm{mL} \cdot \mathrm{cm}^{-2} \cdot \mathrm{min}^{-1}\right)\end{array}$ & Ref. \\
\hline $\mathrm{La}_{0.3} \mathrm{Sr}_{0.7} \mathrm{FeO}_{3-\delta}$ & $3.00^{a}$ & 860 & $17 \% \mathrm{H}_{2} \mathrm{O}(\mathrm{g}) ; \mathrm{He}$ & & 0.005 & {$[76]$} \\
\hline $\mathrm{La}_{0.3} \mathrm{Sr}_{0.7} \mathrm{FeO}_{3-\delta}$ & $3.00^{a}$ & 860 & $17 \% \mathrm{H}_{2} \mathrm{O}(\mathrm{g}) ; \mathrm{CO}$ & & 0.015 & {$[76]$} \\
\hline $\begin{array}{c}\mathrm{La}_{0.7} \mathrm{Sr}_{0.3} \mathrm{Cu}_{0.2} \mathrm{Fe}_{0.8} \mathrm{O}_{3-\delta} \\
\quad(\mathrm{LSCF} 7328)\end{array}$ & 0.53 & 900 & $49 \% \mathrm{H}_{2} \mathrm{O}(\mathrm{g}) ; 80 \% \mathrm{H}_{2}$ & $\mathrm{Pt} / \mathrm{SDC}$ & 1.9 & {$[82]$} \\
\hline LSCF7328 & 0.02 & 900 & $49 \% \mathrm{H}_{2} \mathrm{O}(\mathrm{g}) ; 80 \% \mathrm{H}_{2}$ & - & $\approx 9$ & {$[83]$} \\
\hline LSCF7328 & 0.05 & 900 & $49 \% \mathrm{H}_{2} \mathrm{O}(\mathrm{g}) ; 80 \% \mathrm{H}_{2}$ & $\mathrm{Pt} / \mathrm{SDC}$ & 11.4 & {$[82]$} \\
\hline $\mathrm{Ba}_{0.98} \mathrm{Ce}_{0.05} \mathrm{Fe}_{0.95} \mathrm{O}_{3-\delta}(\mathrm{BCF})$ & 0.50 & 900 & $90 \% \mathrm{H}_{2} \mathrm{O}(\mathrm{g}) ; 80 \% \mathrm{H}_{2}$ & $\begin{array}{c}\mathrm{Ru} / \mathrm{SDC} \\
\left(m_{\mathrm{Ru}} / m_{\text {catalyst }}=1 \%\right)\end{array}$ & 10.2 & [49] \\
\hline $\mathrm{BaZr}_{x} \mathrm{Co}_{y} \mathrm{Fe}_{z} \mathrm{O}_{3-\delta}(\mathrm{BZCF})$ & $0.17^{a}$ & 900 & $75 \% \mathrm{H}_{2} \mathrm{O}(\mathrm{g}) ; 4 \% \mathrm{CH}_{4}$ & - & 2.2 & {$[28]$} \\
\hline $\mathrm{La}_{0.9} \mathrm{Ca}_{0.1} \mathrm{FeO}_{3-\delta}$ (LCF-91) & 0.90 & 990 & $50 \% \mathrm{H}_{2} \mathrm{O}(\mathrm{g}) ; 5 \% \mathrm{CH}_{4}$ & - & 0.5 & {$[31]$} \\
\hline $\mathrm{SrFeCo}_{0.5} \mathrm{O}_{x}(\mathrm{SFC} 2)$ & 1.04 & 900 & $49 \% \mathrm{H}_{2} \mathrm{O}(\mathrm{g}) ; 80 \% \mathrm{H}_{2}$ & 一 & 4.0 & {$[78]$} \\
\hline $\mathrm{SrFeCo}_{0.5} \mathrm{O}_{x}(\mathrm{SFC} 2)$ & 0.02 & 900 & $69 \% \mathrm{H}_{2} \mathrm{O}(\mathrm{g}) ; 80 \% \mathrm{H}_{2}$ & - & 17.4 & {$[78]$} \\
\hline $\mathrm{SrCo}_{0.4} \mathrm{Fe}_{0.5} \mathrm{Zr}_{0.1} \mathrm{O}_{3-\delta}(\mathrm{SCFZ})$ & - & 900 & $69 \% \mathrm{H}_{2} \mathrm{O}(\mathrm{g}) ; 6 \% \mathrm{EtOH}$ & - & 3.4 & [108] \\
\hline
\end{tabular}

${ }^{a}$ The thickness here refers to the wall thickness of the tubular or hollow-fiber membrane.

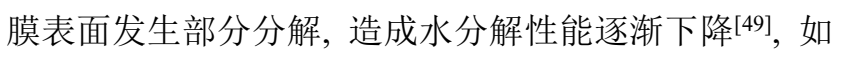
图 3 所示.

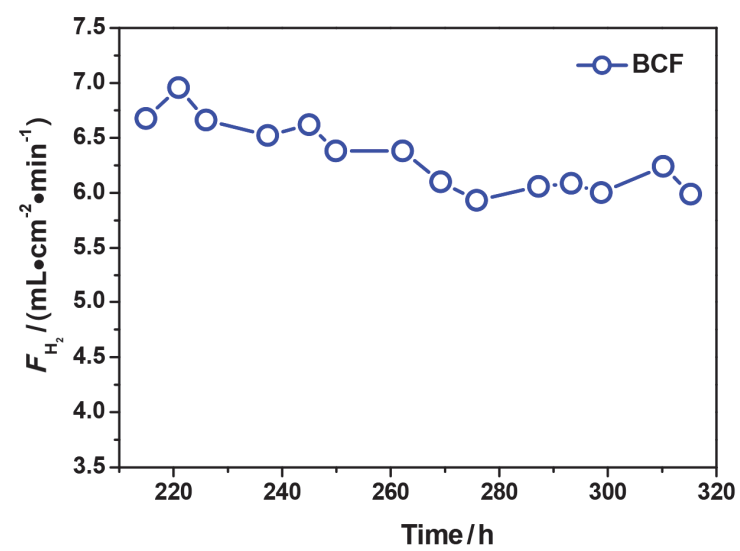

图 $3 \mathrm{BCF}$ 膜材料在 $900{ }^{\circ} \mathrm{C}$ 的水分解稳定性 ${ }^{[49]}$. 膜两侧条件分别是 $90 \% \mathrm{H}_{2} \mathrm{O}$ 和 $50 \% \mathrm{H}_{2}$

Figure 3 Water splitting stability of BCF membrane at $900{ }^{\circ} \mathrm{C}{ }^{[49]} .90 \%$ $\mathrm{H}_{2} \mathrm{O}$ and $50 \% \mathrm{H}_{2}$ fed to the two sides of BCF membrane, respectively. Reprinted with permission from ref. [49], (C) 2016 John Wiley and Sons

稳定性是评价膜反应器能否实际应用的一个重要 指标, 任何原因造成的膜性能衰减均会阻碍其应用. 研 究表明 $1.04 \mathrm{~mm}$ 厚的 $\mathrm{SrFeCo}_{0.5} \mathrm{O}_{x}$ ( $\mathrm{SFC} 2$ )膜在 $900{ }^{\circ} \mathrm{C}$ 的 水分解速率约为 $4.0 \mathrm{~mL} \cdot \mathrm{cm}^{-2} \cdot \mathrm{min}^{-1}{ }^{[78]}$. Lee 等 ${ }^{[78]}$ 制备了 厚度为 $20 \mu \mathrm{m}$ 的 SFC2 薄膜, 在 $900{ }^{\circ} \mathrm{C}$ 时水分解速率达 到 $17.4 \mathrm{~mL} \cdot \mathrm{cm}^{-2} \cdot \mathrm{min}^{-1}$. 虽然该膜材料在 $850{ }^{\circ} \mathrm{C}$ 以上的 水分解速率很高, 但是由于 $\mathrm{SFC} 2$ 材料在 $825 \sim 850{ }^{\circ} \mathrm{C}$ 之间发生相变 ${ }^{[79]}$, 稍低温度下水分解速率急剧降低. $\mathrm{La}_{0.6} \mathrm{Sr}_{0.4} \mathrm{Co}_{0.2} \mathrm{Fe}_{0.8} \mathrm{O}_{3-\delta}(\mathrm{LSCOF})$ 材料在单纯的氧渗透过程 中具有良好的稳定性, 当将管状 $\mathrm{LSCoF}$ 应用在耦合水 分解反应和 POM 反应的膜反应器中时, 在经过约 $400 \mathrm{~h}$ 的测试后, 膜管内表面(POM 反应侧)有部分 $\mathrm{S}$ 和 Co 的 富集, 并出现了针状和球状晶体, 同时膜的表面也出现 了裂缝 ${ }^{[80]} . X$ 射线衍射(XRD)结果显示在水侧(膜管外 侧)膜表面有 $\mathrm{SrO}$ 和 $\mathrm{La}_{2} \mathrm{O}_{3}$ 的析出. 以上结果表明含 $\mathrm{Co}$ 单相膜材料在耦合水分解反应的膜反应器中稳定性较
差.

为了提高膜材料的稳定性, 研究者们开发了多种无 Co 钙钛矿材料应用在水分解反应中 [28,31,76,81-84], 如 $\mathrm{La}_{0.9} \mathrm{Ca}_{0.1} \mathrm{FeO}_{3-\delta} \quad(\mathrm{LCF}-91)^{[31]} 、 \mathrm{La}_{0.7} \mathrm{Sr}_{0.3} \mathrm{Cu}_{0.2} \mathrm{Fe}_{0.8} \mathrm{O}_{3-\delta}$ (LSCF7328) ${ }^{[81-83]}$ 和后面将要详细讨论的双相膜材料等. 但 LCF-91 和 LSCF7328 膜反应器的水分解速率较低, 厚 度为 $0.90 \mathrm{~mm}$ 的 LCF-91 膜在 $990{ }^{\circ} \mathrm{C}$ 的水分解速率只有 $0.5 \mathrm{~mL} \cdot \mathrm{cm}^{-2} \cdot \mathrm{min}^{-1}[31]$. LSCF7328 钙钛矿材料在强还原气 氛中能保持好的结构稳定性 ${ }^{[81]} .0 .53 \mathrm{~mm}$ 厚的 LSCF7328 膜在 $900{ }^{\circ} \mathrm{C}$ 的水分解速率也仅达到 $1.9 \mathrm{~mL} \cdot \mathrm{cm}^{-2}$. $\min ^{-1}{ }^{[82]}$. 降低膜的厚度能减少氧渗透体相扩散阻力, 进而提高膜反应器的水分解速率. 将其制成致密层厚度 为 $50 \mu \mathrm{m}$ 的非对称膜, 在 $900{ }^{\circ} \mathrm{C}$ 时水分解速率达到 11.4 $\mathrm{mL} \cdot \mathrm{cm}^{-2} \cdot \mathrm{min}^{-1}$. 同时, 研究结果表明当水分压从 $3.0 \times$ $10^{3}$ 增加到 $4.9 \times 10^{4} \mathrm{~Pa}$ 时, 水分解速率从 1.4 增加到 10.3 $\mathrm{mL} \cdot \mathrm{cm}^{-2} \cdot \mathrm{min}^{-1}{ }^{[82-83]}$. 这证明了水分压是影响水分解速 率的一个关键因素.

\subsection{2 双相 OTM 材料}

在水分解反应参与的膜反应器中膜两侧的还原性 均较强, 对膜材料的稳定性要求高. 单相 OTM 材料在 水分解反应中稳定性欠佳, 需要发展稳定性更好的膜材 料. 双相膜组成可调, 稳定性较好, 是一类理想的替代 材料. 表 2 列举了一些双相膜材料用于水分解反应时的 性能. 为了提高膜反应器的水分解性能, 在构建双相膜 时要兼顾两相膜材料的离子和电子电导率. 如电子电导 率较低的 $\mathrm{TiO}_{2}-\mathrm{YSZ}$ 双相膜在 $1600{ }^{\circ} \mathrm{C}$ 的水分解速率仅 为 $0.5 \mathrm{~mL} \cdot \mathrm{cm}^{-2} \cdot \mathrm{min}^{-1}[47]$. 使用金属 $\mathrm{Cu}$ 或 $\mathrm{Ni}$ 作为电子导 电相可以弥补双相膜材料电子电导率低的缺陷, 在水分 解反应中表现出良好的性能. 厚度为 $0.46 \mathrm{~mm}$ 的 $\mathrm{Cu}-\mathrm{GDC}$, 在 $900{ }^{\circ} \mathrm{C}$ 的水分解速率为 $2.3 \mathrm{~mL} \cdot \mathrm{cm}^{-2}$ • $\mathrm{min}^{-1}{ }^{[48]}$. Balachandran 等 ${ }^{[85]}$ 设计了 $\mathrm{Ni}$ 作为电子导电相的 $\mathrm{Ni}-\mathrm{GDC}$ 材料, 相同厚度的 Ni-GDC 和 $\mathrm{Cu}-\mathrm{GDC}$ 的水分 解性能相当. 在膜两侧涂覆 Ni-GDC 多孔层来催化膜 
表 2 双相透氧膜材料在 $900{ }^{\circ} \mathrm{C}$ 的水分解性能

Table 2 The water splitting performance of dual-phase oxygen transport membranes at $900{ }^{\circ} \mathrm{C}$

\begin{tabular}{|c|c|c|c|c|c|c|}
\hline Membrane materials & $\begin{array}{c}\text { Ratios of two } \\
\text { phases }\end{array}$ & $\begin{array}{c}\text { Thickness/ } \\
\text { mm }\end{array}$ & $\begin{array}{l}\text { Atmospheres } \\
\text { at both sides }\end{array}$ & Catalysts at water side & $\begin{array}{c}F_{\mathrm{H}_{2}} / \\
\left(\mathrm{mL} \cdot \mathrm{cm}^{-2} \cdot \mathrm{min}^{-1}\right)\end{array}$ & Ref. \\
\hline $\begin{array}{c}\left(\mathrm{ZrO}_{2}\right)_{0.8}\left(\mathrm{TiO}_{2}\right)_{0.1}\left(\mathrm{Y}_{2} \mathrm{O}_{3}\right)_{0.1} \\
\left(\mathrm{TiO}_{2}-\mathrm{YSZ}\right)\end{array}$ & - & $2^{a}$ & $\begin{array}{c}17 \% \mathrm{H}_{2} \mathrm{O}(\mathrm{g}) ; \mathrm{H}_{2} / \mathrm{CO}_{2} \\
\left(\mathrm{P}_{\mathrm{O}_{2}} 10^{-7} \mathrm{~Pa}\right)\end{array}$ & - & $\approx 0.5^{b}$ & [47] \\
\hline $\mathrm{Cu}-\mathrm{GDC}$ & $2 / 3$ (volume ratio) & 0.46 & $49 \% \mathrm{H}_{2} \mathrm{O}(\mathrm{g}) ; 80 \% \mathrm{H}_{2}$ & - & 2.3 & {$[48]$} \\
\hline Ni-GDC & $2 / 3$ (volume ratio) & 0.13 & $49 \% \mathrm{H}_{2} \mathrm{O}(\mathrm{g}) ; 80 \% \mathrm{H}_{2}$ & - & 3.9 & [85] \\
\hline Ni-GDC & $2 / 3$ (volume ratio) & 0.13 & $49 \% \mathrm{H}_{2} \mathrm{O}(\mathrm{g}) ; 80 \% \mathrm{H}_{2}$ & Ni-GDC $\left(V_{\mathrm{Ni}} / V_{\mathrm{GDC}}=40 \%\right)$ & 6 & {$[85]$} \\
\hline Ni-GDC & $2 / 3$ (volume ratio) & 0.46 & $49 \% \mathrm{H}_{2} \mathrm{O}(\mathrm{g}) ; 80 \% \mathrm{H}_{2}$ & - & 2.4 & {$[85]$} \\
\hline $\begin{array}{c}\mathrm{Gd}_{0.2} \mathrm{Ce}_{0.8} \mathrm{O}_{1.9-\delta^{-}} \\
\mathrm{Gd}_{0.08} \mathrm{Sr}_{0.88} \mathrm{Ti}_{0.95} \mathrm{Al}_{0.05} \mathrm{O}_{3 \pm \delta} \\
(\mathrm{GDC}-\mathrm{GSTA})\end{array}$ & $3 / 2$ (volume ratio) & 1.1 & $25 \% \mathrm{H}_{2} \mathrm{O}(\mathrm{g}) ; 50 \% \mathrm{H}_{2}$ & - & 2.0 & [86] \\
\hline GDC-GSTA & $3 / 2$ (volume ratio) & 1.1 & $25 \% \mathrm{H}_{2} \mathrm{O}(\mathrm{g}) ; 50 \% \mathrm{H}_{2}$ & $\mathrm{NiO}-\mathrm{GDC}\left(V_{\mathrm{NiO}} / V_{\mathrm{GDC}}=50 \%\right)$ & 2.6 & [86] \\
\hline GDC-GSTA & $3 / 2$ (volume ratio) & 1.1 & $25 \% \mathrm{H}_{2} \mathrm{O}(\mathrm{g}) ; 100 \% \mathrm{H}_{2}$ & $\mathrm{NiO}-\mathrm{GDC}\left(V_{\mathrm{NiO}} / V_{\mathrm{GDC}}=50 \%\right)$ & 3.1 & [86] \\
\hline GDC-GSTA & $3 / 2$ (volume ratio) & 0.025 & $25 \% \mathrm{H}_{2} \mathrm{O}(\mathrm{g})$ & - & $\approx 10$ & [87] \\
\hline $\begin{array}{c}\mathrm{Ce}_{0.85} \mathrm{Sm}_{0.15} \mathrm{O}_{1.925^{-}} \\
\mathrm{Sm}_{0.6} \mathrm{Sr}_{0.4} \mathrm{Al}_{0.3} \mathrm{Fe}_{0.7} \mathrm{O}_{3-\delta} \\
(\mathrm{SDC}-\mathrm{SSAF})\end{array}$ & 3/1 (mass ratio) & 0.04 & $90 \% \mathrm{H}_{2} \mathrm{O}(\mathrm{g}) ; 80 \% \mathrm{H}_{2}$ & $\mathrm{Ru} / \mathrm{SDC}\left(m_{\mathrm{Ru}} / m_{\text {catalyst }}=1 \%\right)$ & 15.5 & {$[27]$} \\
\hline SDC-SSAF & 3/1 (mass ratio) & 0.40 & $90 \% \mathrm{H}_{2} \mathrm{O}(\mathrm{g}) ; 80 \% \mathrm{H}_{2}$ & $\mathrm{Ru} / \mathrm{SDC}\left(m_{\mathrm{Ru}} / m_{\text {catalyst }}=1 \%\right)$ & 5.0 & [98] \\
\hline $\begin{array}{c}\mathrm{Ce}_{0.85} \mathrm{Sm}_{0.15} \mathrm{O}_{1.925^{-}} \\
\mathrm{Sm}_{0.6} \mathrm{Sr}_{0.4} \mathrm{Cr}_{0.3} \mathrm{Fe}_{0.7} \mathrm{O}_{3-\delta} \\
(\mathrm{SDC}-\mathrm{SSCF})\end{array}$ & 3/1 (mass ratio) & 0.70 & $90 \% \mathrm{H}_{2} \mathrm{O}(\mathrm{g}) ; 80 \% \mathrm{H}_{2}$ & $\mathrm{Ni} / \mathrm{SDC}\left(m_{\mathrm{Ni}} / m_{\mathrm{SDC}}=10 \%\right)$ & 5.3 & {$[51]$} \\
\hline SDC-SSCF & 3/1 (mass ratio) & 0.50 & $90 \% \mathrm{H}_{2} \mathrm{O}(\mathrm{g}) ; 80 \% \mathrm{H}_{2}$ & $\mathrm{Ni} / \mathrm{SDC}\left(m_{\mathrm{Ni}} / m_{\mathrm{SDC}}=10 \%\right)$ & 6.5 & [51] \\
\hline SDC-SSCF & 3/1 (mass ratio) & 0.36 & $90 \% \mathrm{H}_{2} \mathrm{O}(\mathrm{g}) ; 80 \% \mathrm{H}_{2}$ & $\mathrm{Ni} / \mathrm{SDC}\left(m_{\mathrm{Ni}} / m_{\mathrm{SDC}}=10 \%\right)$ & 6.9 & {$[51]$} \\
\hline SDC-SSCF & 3/1 (mass ratio) & 0.36 & $90 \% \mathrm{H}_{2} \mathrm{O}(\mathrm{g}) ; 80 \% \mathrm{H}_{2}$ & $\mathrm{Ni} / \mathrm{SDC}\left(m_{\mathrm{Ni}} / m_{\mathrm{SDC}}=10 \%\right)$ & 4.6 & [51] \\
\hline SDC-SSCF & 3/1 (mass ratio) & 0.36 & $90 \% \mathrm{H}_{2} \mathrm{O}(\mathrm{g}) ; 80 \% \mathrm{H}_{2}$ & $\mathrm{Ni} / \mathrm{SDC}\left(m_{\mathrm{Ni}} / m_{\mathrm{SDC}}=10 \%\right)$ & 6.2 & [51] \\
\hline $\begin{array}{l}\mathrm{Ce}_{0.85} \mathrm{Sm}_{0.15} \mathrm{O}_{1.925^{-}} \\
\mathrm{Sr}_{2} \mathrm{Fe}_{1.5} \mathrm{Mo}_{0.5} \mathrm{O}_{6-\delta} \\
\quad(\mathrm{SDC}-\mathrm{SFM})\end{array}$ & $7 / 3$ (mass ratio) & 0.5 & $90 \% \mathrm{H}_{2} \mathrm{O}(\mathrm{g}) ; 80 \% \mathrm{H}_{2}$ & $\mathrm{Ni} / \mathrm{SDC}\left(m_{\mathrm{Ni}} / m_{\mathrm{SDC}}=10 \%\right)$ & 6.3 & [50] \\
\hline SDC-SSCF & 3/1 (mass ratio) & 0.04 & $90 \% \mathrm{H}_{2} \mathrm{O}(\mathrm{g}) ; 80 \% \mathrm{H}_{2}$ & $\mathrm{Ni} / \mathrm{SDC}\left(m_{\mathrm{Ni}} / m_{\mathrm{SDC}}=2 \%\right)$ & 15.8 & {$[52]$} \\
\hline SDC-SSCF & 3/1 (mass ratio) & 0.04 & $90 \% \mathrm{H}_{2} \mathrm{O}(\mathrm{g}) ; 80 \% \mathrm{H}_{2}$ & $\begin{array}{c}(\mathrm{Ni}+\mathrm{Ru}) / \mathrm{SDC}\left(m_{\mathrm{Ni}} / m_{\mathrm{SDC}}=\right. \\
\left.1 \%, m_{\mathrm{Ru}} / m_{\mathrm{SDC}}=1 \%\right)\end{array}$ & 19.3 & {$[52]$} \\
\hline $\begin{array}{c}\mathrm{Ce}_{0.9} \mathrm{Pr}_{0.1} \mathrm{O}_{2-\delta^{-}} \\
\mathrm{Pr}_{0.1} \mathrm{Sr}_{0.9} \mathrm{Mg}_{0.1} \mathrm{Ti}_{0.9} \mathrm{O}_{3-\delta} \\
\quad(\mathrm{CPO}-\mathrm{PSMTi})\end{array}$ & $3 / 2$ (molar ratio) & 0.70 & $88 \% \mathrm{H}_{2} \mathrm{O}(\mathrm{g}) ; 40 \% \mathrm{H}_{2}$ & - & 0.41 & [32] \\
\hline
\end{tabular}

${ }^{a}$ The thickness here refers to the wall thickness of the tubular membrane; ${ }^{b}$ The water splitting rate here is evaluated at $1600{ }^{\circ} \mathrm{C}$.

两侧反应, 并将膜厚度降至 $0.13 \mathrm{~mm}$ 时, Ni-GDC 的水分 解速率可提升至 $6.0 \mathrm{~mL} \cdot \mathrm{cm}^{-2} \bullet \mathrm{min}^{-1}[85]$. 为了进一步提高 双相膜反应器中的水分解速率, 研究者们更加关注氧离 子导体-混合导体双相膜材料的设计. 如 $1.1 \mathrm{~mm}$ 厚的 $\mathrm{Gd}_{0.2} \mathrm{Ce}_{0.8} \mathrm{O}_{1.9-\delta}-\mathrm{Gd}_{0.08} \mathrm{Sr}_{0.88} \mathrm{Ti}_{0.95} \mathrm{Al}_{0.05} \mathrm{O}_{3 \pm \delta}$ (GDC-GSTA) 双 相膜在 $900{ }^{\circ} \mathrm{C}$ 的水分解速率为 $3.1 \mathrm{~mL} \cdot \mathrm{cm}^{-2} \cdot \mathrm{min}^{-1}[86]$. 为 了提高膜反应器的水分解性能, Wang 等 ${ }^{[87]}$ 将 GDC-GSTA 制备成 $25 \mu \mathrm{m}$ 厚的非对称负载膜, 在 $900{ }^{\circ} \mathrm{C}$ 的水分解速率达到 $10 \mathrm{~mL} \cdot \mathrm{cm}^{-2} \cdot \mathrm{min}^{-1}$.

当体相扩散是决速步时, 氧渗透过程主要受电导率 和氧分压梯度的影响. 很多文献已经报道了 $\mathrm{La}_{1-x} \mathrm{Sr}_{x} \mathrm{FeO}_{3-\delta}{ }^{[88-91]}$ 和 $\mathrm{La}_{1-x} \mathrm{Sr}_{x} \mathrm{M}_{1-y} \mathrm{Fe}_{y} \mathrm{O}_{3-\delta}(\mathrm{M}=\mathrm{Al}, \mathrm{Ti}, \mathrm{Cr}$ 等 $)^{[89,92-96]}$ 这些钙钛矿材料的总电导率在高氧分压范围 内 $\left(10^{-5} \mathrm{~Pa}\right)$ 主要是 $\mathrm{p}$-型电子电导, 在低的氧分压范围内 $\left(10^{-10} \mathrm{~Pa}\right)$ 主要是 $\mathrm{n}$-型电子电导. 在高温下, 在中低氧分 压范围内(通常是 $10^{-5} \sim 10^{-10} \mathrm{~Pa}$ ) 电导率会出现一个最小 值. 在这一点, 氧离子电导占主导, $p$-型和 $n$-型电子电导 的贡献相当, 均比较小, 如图 4 所示 ${ }^{[97]}$. 另外, 文献报 道的 $\mathrm{Sm}_{0.6} \mathrm{Sr}_{0.4} \mathrm{Al}_{0.3} \mathrm{Fe}_{0.7} \mathrm{O}_{3-\delta}(\mathrm{SDC}-\mathrm{SSAF})$ 在中低氧分压范 围内的电子电导也非常低 ${ }^{[97]}$. 在 POM 膜反应器中混合
导体的电导率在 I 区域随氧分压的减小而减小, 在 II 区 域达到最低值，在 III 区域随氧分压的减小而增大. 尽管 混合导体材料在氧化和强还原气氛下的电子电导率均 很高, 但是应用在氧分压梯度很大, 尤其跨越中低氧分 压条件的领域时, 它在中低氧分压下(II 区)的电子电导 不足, 可能是限制其透氧性能的关键因素. 在水分解反 应参与的 OTM 反应器中膜两侧的氧分压通常在 $10^{-7} \sim$ $10^{-16} \mathrm{~Pa}$ ，也存在中低氧分压的区域. 所以，混合导体在 中低氧分压下(II 区)的电子电导不足限制了其透氧性能, 进而影响水分解性能. 提高材料在中低氧分压条件下的 电子电导率是提升膜渗透性的关键. 在 $\mathrm{CeO}_{2}$ 基双相膜 中, $\mathrm{CeO}_{2}$ 是主相, 主要提供氧离子电导. $\mathrm{CeO}_{2}$ 的氧离子 电导率受氧分压的影响不大, 在中低氧分压时 $\mathrm{CeO}_{2}$ 还 不能被还原产生足够的 $\mathrm{n}$-型电子电导. 所以, 双相膜材 料中的混合导体相在中低氧分压下的电子电导不足严 重影响氧渗透性能, 进而影响水分解性能. 因此, 可以 通过优化双相膜中的混合导体相的组成来调节其在中 低氧分压下的电子导电性能来获得较高的水分解性能. Cai 等 ${ }^{[97]}$ 利用 $\mathrm{Cr} 、 \mathrm{Fe}$ 共掺杂的 $\mathrm{Sm}_{0.6} \mathrm{Sr}_{0.4} \mathrm{Cr}_{0.3} \mathrm{Fe}_{0.7} \mathrm{O}_{3-\delta}$ (SSCF) 作为双相膜中的混合导体相, 制备 
$\mathrm{Ce}_{0.85} \mathrm{Sm}_{0.15} \mathrm{O}_{1.925}-\mathrm{Sm}_{0.6} \mathrm{Sr}_{0.4} \mathrm{Cr}_{0.3} \mathrm{Fe}_{0.7} \mathrm{O}_{3-\delta}(\mathrm{SDC}-\mathrm{SSCF}$ )双相 膜, 在中低氧分压下产生可观的电子电导率, 来增强水 分解反应性能. $0.50 \mathrm{~mm}$ 厚的 SDC-SSCF 在 $900{ }^{\circ} \mathrm{C}$ 的水 分解速率约 $7.0 \mathrm{~mL} \cdot \mathrm{cm}^{-2} \cdot \mathrm{min}^{-1}[51]$, 大于在相同测试条件 下 $0.40 \mathrm{~mm}$ 厚的 $\mathrm{Ce}_{0.85} \mathrm{Sm}_{0.15} \mathrm{O}_{1.925}-\mathrm{Sm}_{0.6} \mathrm{Sr}_{0.4} \mathrm{Al}_{0.3} \mathrm{Fe}_{0.7} \mathrm{O}_{3-\delta}$ $(\mathrm{SDC}-\mathrm{SSAF})$ 膜的水分解速率 $\left(5.2 \mathrm{~mL} \cdot \mathrm{cm}^{-2} \cdot \mathrm{min}^{-1}\right)^{[98]}$.

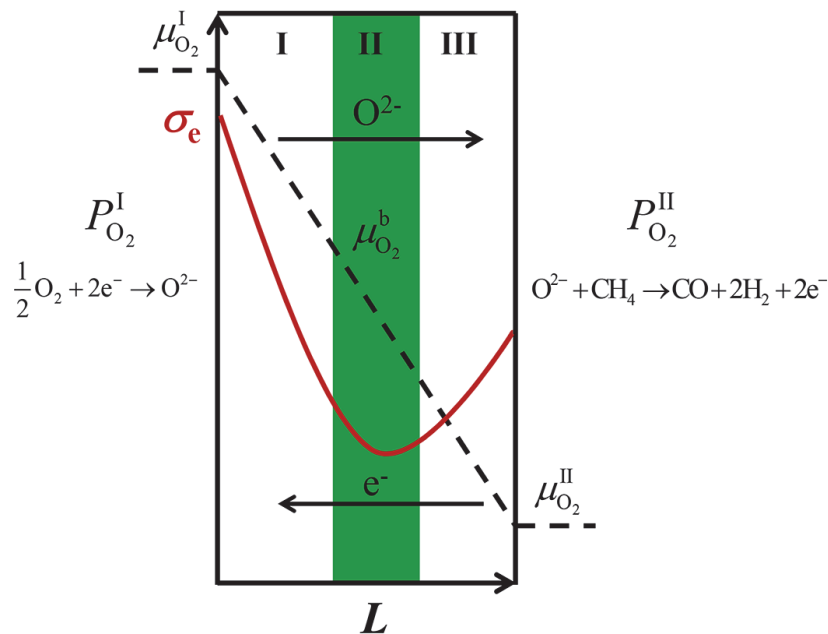

图 4 POM 膜反应器中膜两侧的氧化学势和电导率变化示意图 ${ }^{[97]}$. 其 中 $L$ 代表膜的厚度, $\sigma_{\mathrm{e}}$ 代表电子电导率

Figure 4 Schematic illustration of oxygen chemical potential drop and conductivity change across an OTM membrane in the POM reactor, where $L$ represents the thickness of membrane, and $\sigma_{\mathrm{e}}$ is the electronic conductivity ${ }^{[97]}$. Reprinted with permission from ref. [97], (C) 2016 Elsevier

目前, 工业上 $90 \%$ 以上的氢气来自于化石燃料的转 化 ${ }^{[99-101]}$. 这些方法制备的氢气中不可避免的含有 CO、

$\mathrm{CO}_{2} 、 \mathrm{H}_{2} \mathrm{~S}$ 等杂质, 这些杂质对膜材料存在明显的毒化 作用. 所以为了进一步提高膜材料的稳定性, 需要制备 能在含有 $\mathrm{CO}_{2}$ 和 $\mathrm{H}_{2} \mathrm{~S}$ 等杂质的苛刻气氛中长时间稳定运 行的膜材料. $\mathrm{Li}$ 和 $\mathrm{Cai}$ 等设计了在氧化还原气氛下均有 较高电导率和良好稳定性的 SDC-SSAF ${ }^{[27] 、}$ $\mathrm{Ce}_{0.85} \mathrm{Sm}_{0.15} \mathrm{O}_{1.925}-\mathrm{Sr}_{2} \mathrm{Fe}_{1.5} \mathrm{Mo}_{0.5} \mathrm{O}_{6-\delta} \quad$ (SDC-SFM) [50] 和 SDC-SSCF [53]双相膜, 它们在含 $\mathrm{CO}_{2}$ 和 $\mathrm{H}_{2} \mathrm{~S}$ 杂质的还原
性气氛中能保持长期的稳定性，如图 5 所示.

$\mathrm{Jia}$ 等 ${ }^{[32]}$ 设计合成了不易被过度还原的含 $\mathrm{Ti}$ 钻钛矿 材料的 $\mathrm{Ce}_{0.9} \mathrm{Pr}_{0.1} \mathrm{O}_{2-\delta}-\mathrm{Pr}_{0.1} \mathrm{Sr}_{0.9} \mathrm{Mg}_{0.1} \mathrm{Ti}_{0.9} \mathrm{O}_{3-\delta}$ (CPO-PSMTi) 双相膜, 但 $0.70 \mathrm{~mm}$ 厚的 CPO-PSMTi 双相膜在 $900{ }^{\circ} \mathrm{C}$ 的水分解速率仅为 $0.41 \mathrm{~mL} \cdot \mathrm{cm}^{-2} \cdot \mathrm{min}^{-1}$. 在以后的工作 中仍需要设计水分解速率高和稳定性好的膜反应器.

\section{2 影响膜材料性能的因素}

OTM 反应器中的水分解反应性能受膜材料的氧渗 透性能的限制, 尤其当膜的体相阻力占比较大时, 体相 扩散将成为限制水分解性能的关键. 根据材料的理化性 质, 设计出有较高透氧量的材料是研究的重点. 在传统 的透氧过程中, 膜两侧所处的氧分压均较高 $\left(1 \sim 10^{5} \mathrm{~Pa}\right)$, 而在水分解反应条件下, 膜两侧均处在较低氧分压下, 这两种条件下膜材料的电导率不同. 根据 Wagner 方程得知 $\left(J_{\mathrm{O}_{2}}=\frac{R T}{16 F^{2} L} \int_{\ln P_{\mathrm{O}_{2}}^{\mathrm{l}}}^{\ln P_{2}^{\mathrm{h}}} \frac{\sigma_{\mathrm{e}} \sigma_{\mathrm{i}}}{\sigma_{\mathrm{e}}+\sigma_{\mathrm{i}}} d \ln P_{\mathrm{O}_{2}}, \sigma_{\mathrm{e}}\right.$ 和 $\sigma_{\mathrm{i}}$ 分别代表 $\mathrm{OTM}$ 材料的电子电导率和氧离子电导率, $L$ 为膜的厚度, $P_{\mathrm{O}_{2}}^{\mathrm{h}}$ 和 $P_{\mathrm{O}_{2}}^{\mathrm{l}}$ 分别为高氧分压侧和低氧分压侧的氧分压), 膜厚度、电导率、氧分压和温度等是影响氧渗透过程的 关键因素，进而影响水分解速率. 这些因素对水分解性 能的影响总结如下.

\subsection{1 膜厚度的影响}

若膜材料组成不变, 通过降低膜厚度来减小体相扩 散阻力是一种最常见的提高氧渗透性能或水分解速率 的方法. 对 Ni-GDC 膜材料来说, 当膜厚从 1.70 降低到 $0.10 \mathrm{~mm}$ 时, 水分解速率从 1.2 增加到 $4.3 \mathrm{~mL} \cdot \mathrm{cm}^{-2}$ • $\mathrm{min}^{-1}$; 当膜厚从 0.28 降至 $0.13 \mathrm{~mm}$ 时, 水分解速率增加 缓慢, 虽然厚度降低了一半, 但水分解速率仅增加了 $18 \%$ (从 3.3 仅增加到 $\left.3.9 \mathrm{~mL} \cdot \mathrm{cm}^{-2} \cdot \mathrm{min}^{-1}\right)^{[85]}$. 假设体相扩 散是决速步，水分解速率应该增加 1 倍，这说明随着厚 度的降低，表面反应的影响逐渐加大．当在 $0.28 \mathrm{~mm}$ a

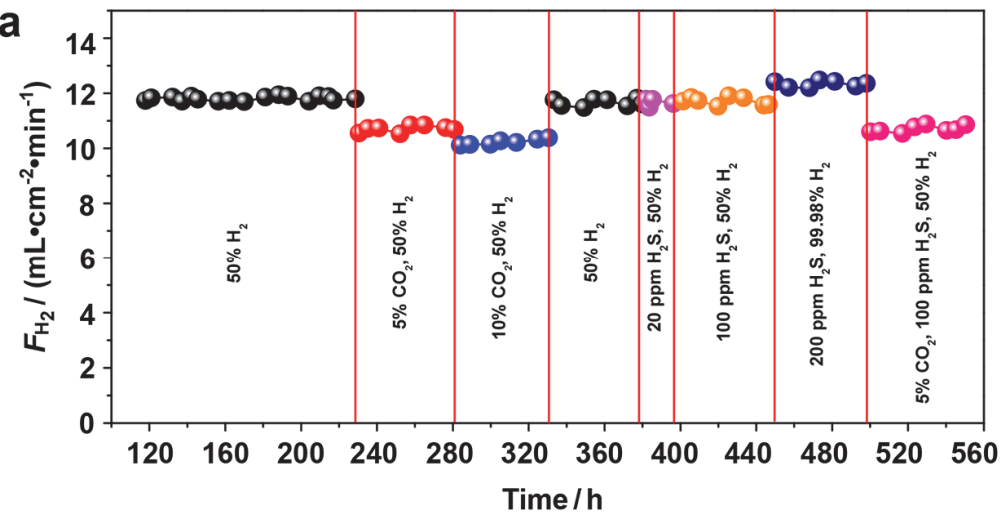

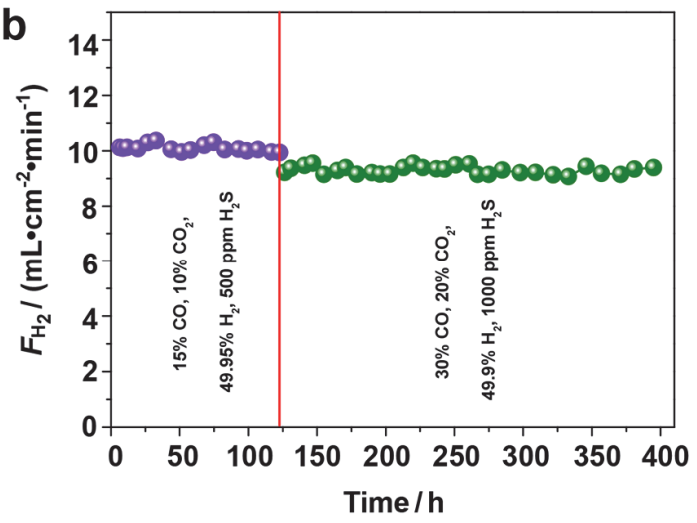

图 5 SDC-SSCF 双相膜在 $900{ }^{\circ} \mathrm{C}$ 不同气氛下的水分解稳定性 ${ }^{[48]}$. 水侧条件为 $90 \% \mathrm{H}_{2} \mathrm{O}$, 使用的不同还原性气体标注在图中

Figure 5 The stability of SDC-SSCF dual-phase membrane under different atomospheres at $900{ }^{\circ} \mathrm{C}[48]$. The condition at water side is $90 \% \mathrm{H}_{2} \mathrm{O}$, and the different reducing gases used are marked in the figure. Reprinted with permission from ref. [48], (C) 2020 John Wiley and Sons 
和 $0.13 \mathrm{~mm}$ 厚的 Ni-GDC 膜表面增加 Ni-GDC 多孔层时, 水分解速率分别增加到 4.6 和 $6.0 \mathrm{~mL} \cdot \mathrm{cm}^{-2} \cdot \mathrm{min}^{-1}{ }^{[85]}$. 膜 表面的多孔层增加了表面反应速率, 进而增加了水分解 速率.

在研究不同厚度的 SDC-SSCF 膜材料的水分解性 能时, Cai 等[51]对耦合水分解反应和氢氧化反应的膜反 应器建立了简单模型, 定量计算了不同温度下水分解反 应和氢氧化反应的表面反应阻力 $\left(r^{\mathrm{s}}\right)$ 、膜的体相扩散阻力 $\left(r^{\mathrm{b}}\right)$, 如图 6 所示. 由于用于模型计算的数据有限, 所以, 使用 $r^{\mathrm{s}}$ 表示水分解反应阻力和氢氧化反应阻力的总和. 总阻力 $\left(r^{\mathrm{tot}}\right)$ 可以由 $r^{\mathrm{b}}$ 和 $r^{\mathrm{s}}$ 的和得到 $\left(r^{\mathrm{tot}}=r^{\mathrm{s}}+r^{\mathrm{b}}\right)$. 另外,

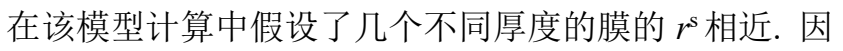
为三个不同厚度的膜反应器在相同温度的水侧的氧分 压 $\left(10^{-8} \sim 10^{-10} \mathrm{~Pa}\right)$ 和还原气体侧的氧分压 $\left(10^{-13} \sim 10^{-16}\right.$ $\mathrm{Pa})$ 分别处在相同的数量级, 改变不大. 而且, 水侧和还 原气体侧的氧分压梯度很高 $\left(10^{5} \sim 10^{6}\right)$. 所以, 假设三 个不同厚度的膜的 $r^{\mathrm{s}}$ 相近是合理的. 如图 6 所示, 随着 温度的增加, $r^{\mathrm{b}}$ 和 $r^{\mathrm{tot}}$ 均降低. $r^{\mathrm{b}}$ 在某一温度下随着厚度 的增加而线性增加. $r^{\mathrm{b} /} r^{\mathrm{tot}}$ 的比值反映了水分解速率受体 相扩散控制的程度. 增加温度和膜厚度将会增加体相扩 散阻力在总阻力中的占比, 或者减小表面反应阻力的占 比. 对 $0.70 \mathrm{~mm}$ 厚的 SDC-SSCF 膜来说, 在所有测试温 度下 $r^{\mathrm{b} /} / r^{\mathrm{tot}}$ 的比值均高于 $50 \%$. 这意味着在所研究的温 度范围内 $r^{\mathrm{b}}$ 比 $r^{\mathrm{s}}$ 大. 但是, 对 $0.36 \mathrm{~mm}$ 厚的 SDC-SSCF 膜来说, $r^{\mathrm{s}}$ 比 $r^{\mathrm{b}}$ 稍大. 考虑到 $r^{\mathrm{b}}$ 和 $r^{\mathrm{s}}$ 相差不大, 在所测试 的厚度范围内, 水分解过程由体相扩散和表面反应共同 控制. 可以推测, 当膜的厚度进一步降低, 表面反应将 是膜反应器中限制水分解过程的决速步 [51].

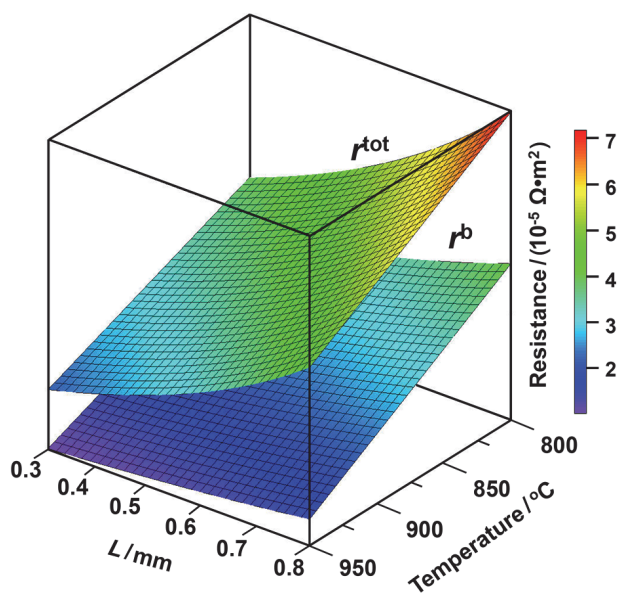

图 6 总阻力 $\left(r^{\mathrm{tot}}\right)$ 和体相阻力 $\left(r^{\mathrm{b}}\right)$ 对温度和膜厚度的关系图 ${ }^{[51]}$

Figure 6 Dependence of the total resistance $\left(r^{\text {tot }}\right)$ and bulk resistance $\left(r^{\mathrm{b}}\right)$ on temperature and membrane thickness $(L)^{[51]}$. Reprinted with permission from ref. [51], (C) 2019 Elsevier

对于具有薄致密层的非对称膜, 其厚多孔层为薄致 密层提供机械支撑, 但是多孔支撑层的孔隙率以及孔径 大小影响气体扩散, 产生的浓差极化效应会降低膜的分 离性能 ${ }^{[3,102-106]}$, 进而影响水分解性能. 因此, 可通过优
化多孔层的结构来实现气体快速输送和提高分离性能. Cai 等 ${ }^{[107]}$ 通过流延法和激光刻槽技术得到在多孔层具 有直通槽的非对称 SDC-SSCF 膜, 如图 7 所示. 该膜反 应器的水分解性能得到约 $30 \%$ 的提升. 目前 OTM 反应 器中涉及多孔层优化的研究还相对较少. 仅靠降低膜厚 度已不能大幅提高水分解性能，发展具有较高催化活性 的水分解催化剂和氧移除反应催化剂将具有重要意义. 后续对于水分解催化剂的研究将在第 4 节详细介绍.

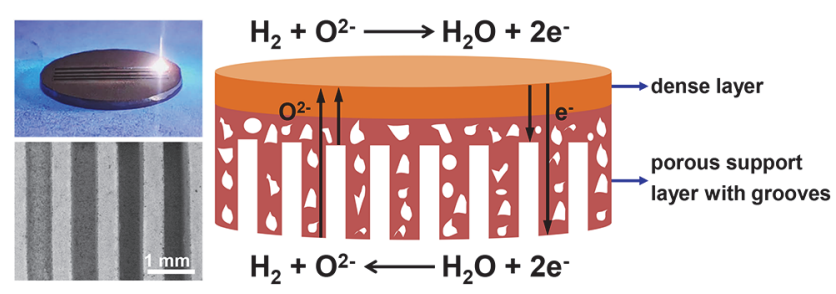

图 7 激光刻槽技术制备多孔层具有直通槽的非对称膜 ${ }^{[107]}$

Figure 7 Asymmetric membranes with straight grooves in the porous support layer were prepared by laser grooving technique ${ }^{[107]}$

\section{2 .2 电导率和温度}

膜两侧的氧分压梯度影响膜材料电导率和氧渗透 过程, 这些因素进一步影响膜反应器的水分解性能. 在 构建双相膜时要兼顾两相膜材料的离子和电子电导率. 如电子电导率较低的 $\mathrm{TiO}_{2}$-YSZ 双相膜的水分解速率也 非常低 ${ }^{[47]}$. 使用金属 $\mathrm{Cu}$ 或 $\mathrm{Ni}$ 作为电子导电相可以弥补 双相膜材料电子电导率低的缺陷，在水分解反应中表现 出良好的性能. 另外, 本综述在双相膜材料优化方面已 经提及通过改变膜材料在中低氧分压下的电导性能来 提高膜反应器的水分解性能, 如在 3.1.2 节介绍的 SDC-SSCF 和 SDC-SFM 双相膜在膜两侧所处的氧分压 条件下均有较高的电导率, 相应的它们的水分解速率也 高于受电子电导限制的 SDC-SSAF 双相膜 [50-51,98].

温度是影响膜材料氧渗透过程的另一重要因素. 虽 然随着温度增加, 氧分压梯度或者相应的驱动力略微降 低, 但是水分解膜反应器中的一些关键步骤, 如水的分 解、氧离子和电子的偶极输运以及 POM 或氢氧化反应 都是热活化的. 相关的反应速率和扩散速率均随温度的 升高而增大. 对于 $0.36 \mathrm{~mm}$ 厚的 SDC-SSCF 膜, 当温度 从 $800{ }^{\circ} \mathrm{C}$ 增加到 $940{ }^{\circ} \mathrm{C}$, 相应的水分解速率从 4.2 增加 到 $7.7 \mathrm{~mL} \cdot \mathrm{cm}^{-2} \cdot \mathrm{min}^{-1}[51]$. 水分解速率随温度的增加而增 加这一结果表明温度相较于氧分压对水分解速率的影 响更大.

\section{2 .3 氧分压梯度}

膜两侧分别发生水分解反应和氧移除反应，这两侧 的反应速率是影响膜反应器的水分解性能的重要因素. 水分解反应侧的氧分压的影响将在第 4 节中介绍, 这一 部分将总结不同氧移除反应的氧分压梯度对反应性能 的影响.

正如前面提到的不同还原性气体如 $\mathrm{H}_{2} 、 \mathrm{CO} 、 \mathrm{CH}_{4}$ 、 
$\mathrm{C}_{2} \mathrm{H}_{6}$ 等对氧移除反应活性不同，进而造成膜材料的氧 渗透性不同. 图 1 展示了四种不同氧移除反应的水分解 催化膜反应器 ${ }^{[22,28,53,108]}$. 当氧移除反应是合成气时, 将 其和整体煤气化联合循环发电系统(IGCC)耦合可以实 现产氢和 $\mathrm{CO}_{2}$ 捕获 ${ }^{[53]}$. 在 $\mathrm{BCF}$ 膜反应器中, 作者认为 $\mathrm{CO}$ 与氧反应的活性比 $\mathrm{H}_{2}$ 的低, 同时产生的 $\mathrm{CO}_{2}$ 部分占 据催化剂的活性位点, 导致氢氧化反应的活性位点数目 减少 ${ }^{[49]}$. 在 BCFZ 中空纤维膜反应器中, 当用惰性气体 移除渗透过来的氧时, 在 $900{ }^{\circ} \mathrm{C}$ 几乎检测不到生成的 $\mathrm{H}_{2}{ }^{[28-29]}$. 当氧移除反应分别是 $\mathrm{POM}^{[28]}$ 和乙烷氧化脱氢 制乙烯 ${ }^{[22]}$ 时, 在 $800{ }^{\circ} \mathrm{C}$ 的水分解速率分别为 0.7 和 1.2 $\mathrm{mL} \cdot \mathrm{cm}^{-2} \bullet \mathrm{min}^{-1}$. Zhu 等 ${ }^{[108]}$ 在 $\mathrm{SrCo}_{0.4} \mathrm{Fe}_{0.5} \mathrm{Zr}_{0.1} \mathrm{O}_{3-\delta}(\mathrm{SCFZ})$ 管状膜反应器中利用乙醇氧化水蒸汽重整反应实现原 位氧移除, 在 $900{ }^{\circ} \mathrm{C}$ 时水分解速率达到 $3.4 \mathrm{~mL} \cdot \mathrm{cm}^{-2}$ • $\mathrm{min}^{-1}$. 以上结果均证实水分解速率极大地依赖氧移除 速率.

另外, 增加还原气体的浓度即降低氧分压来增加氧 分压梯度, 也能增加水分解速率. 如 $0.90 \mathrm{~mm}$ 厚的 LCF-91 膜, 当还原侧 $\mathrm{CH}_{4}$ 浓度从 $0 \%$ 增加到 5\%, 相应的 水分解速率从 0.008 增加到 $0.50 \mathrm{~mL} \cdot \mathrm{cm}^{-2} \cdot \mathrm{min}^{-1}$ [31]. 对于 $0.36 \mathrm{~mm}$ 厚的 SDC-SSCF 膜在 $900{ }^{\circ} \mathrm{C}$ 时, 当还原侧氢气 浓度从 $10 \%$ 增加到 $100 \%$, 相应的水分解速率从 4.4 增加 到 $7.5 \mathrm{~mL} \cdot \mathrm{cm}^{-2} \cdot \mathrm{min}^{-1}$. 同时, 实验结果也表明改变还原 性气体的流速也能增加氧分压梯度, 进而增加水分解速 率 ${ }^{[51]}$.

选用合适的催化剂将会加速氧移除反应，降低氧移 除反应对水分解性能的限制. LCF-91 膜反应器的 POM 侧在无催化剂和使用 $\mathrm{LCF}+\mathrm{Ni}$ 多孔层催化剂时, 相应 的水分解速率从 0.001 增加到 $0.5 \mathrm{~mL} \cdot \mathrm{cm}^{-2} \cdot \mathrm{min}^{-1}{ }^{[31]}$. SDC-SSCF 非对称膜反应器在氢氧化反应侧使用 $\mathrm{Ru} / \mathrm{SDC}$ 催化剂时的水分解速率较使用 $\mathrm{Ni} / \mathrm{SDC}$ 催化剂 提高了 $80 \%{ }^{[52]}$. 这些结果都表明选用高活性催化剂来 加速氧移除速率能增加水分解速率. 目前对氧移除侧的 催化剂研究还较少, 需要在以后的工作中进一步加强.

\section{4 水分解反应催化剂}

水分解是一个不仅受热力学限制, 而且受动力学限 制的过程. 膜反应器可以突破热力学限制, 而催化剂则 对其动力学性能的影响至关重要. 早期, Park 等 ${ }^{[83]}$ 制备 的 LSCF7328、Wang 等 ${ }^{[86-87]}$ 制备的 GDC-GSTA 以及 Jiang 等[28]制备的 BZCF 膜反应器中, 水分解侧均没有 使用催化剂. 虽然他们将 BZCF 制成 50 $100 \mu \mathrm{m}$ 厚的 中空纤维膜, 水分解速率在 $900{ }^{\circ} \mathrm{C}$ 也仅为 $2.2 \mathrm{~mL} \cdot \mathrm{cm}^{-2}$. $\mathrm{min}^{-1}$. Balachandran 等 ${ }^{[85]}$ 在 $0.13 \mathrm{~mm}$ 厚的 Ni-GDC 膜表 面引入 $\mathrm{Ni}-\mathrm{GDC}$ 多孔层后，水分解速率提高了 70\% (从 3.9 增加到 $6.0 \mathrm{~mL} \cdot \mathrm{cm}^{-2} \cdot \mathrm{min}^{-1}$ ). 他们认为多孔层增加了 表面反应动力学进而增加了水分解速率. 同样, Hong 等 ${ }^{[86]}$ 在 $1.1 \mathrm{~mm}$ 厚的 GDC-GSTA 表面涂覆 Ni-GDC 催化
剂, 水分解速率从 2.0 增加到 $2.6 \mathrm{~mL} \cdot \mathrm{cm}^{-2} \cdot \mathrm{min}^{-1}$.

Cai 等 ${ }^{[51]}$ 认识到催化剂对水分解反应的重要性，在 催化剂设计方面做了大量工作. 如设计了多种非贵金属 催化剂 $\mathrm{M} / \mathrm{SDC}\left(\mathrm{M}=\mathrm{Fe}, \mathrm{Co}, \mathrm{Ni}, m_{\mathrm{M}} / m_{\mathrm{SDC}}=10 \%\right)$, 将它 们涂覆在 $0.36 \mathrm{~mm}$ 厚的 SDC-SSCF 膜的两侧. 在 $900{ }^{\circ} \mathrm{C}$ 相应的水分解速率分别达到 5.0、6.8 和 $7.5 \mathrm{~mL} \cdot \mathrm{cm}^{-2}$. $\mathrm{min}^{-1}$, 也即催化活性 $\mathrm{Co} / \mathrm{SDC}<\mathrm{Fe} / \mathrm{SDC}<\mathrm{Ni} / \mathrm{SDC}$. 同时, 考察了在使用 $\mathrm{Ni} / \mathrm{SDC}$ 催化剂时不同膜厚度的影响，尽 管膜的厚度降低了 $94 \%$ (从 0.70 降至 $0.36 \mathrm{~mm}$ ), 但是在 $900{ }^{\circ} \mathrm{C}$ 相应的水分解速率仅增加了 $33 \%$ (从 4.8 增加到 $\left.6.4 \mathrm{~mL} \cdot \mathrm{cm}^{-2} \cdot \mathrm{min}^{-1}\right)$. 如果膜两侧的表面反应阻力可以忽 略, 或催化剂对水分解反应和氢氧化反应有足够高的催 化活性, 氢分离速率应该增加 $94 \%$. 但是, 实际增加了 $33 \%$, 远低于计算的理论值. 所以，膜两侧的反应在整 个氢分离过程起很重要的作用. 即水分解过程中, $\mathrm{Ni} / \mathrm{SDC}$ 催化剂的催化活性还不够高.

$\mathrm{Cai}$ 等 ${ }^{[51]}$ 定量计算出不同厚度的 SDC-SSCF 膜在不 同温度的体相扩散阻力和表面反应阻力, 如图 6 所示. 结果表明, $0.36 \mathrm{~mm}$ 厚的 SDC-SSCF 膜在 $900{ }^{\circ} \mathrm{C}$ 的表面 反应阻力占比 $51 \%$. 如果降低膜的厚度, 表面反应阻力 的占比将进一步增大, Ni/SDC 催化剂的活性还有待提 高. 为了获得较高的水分解速率, 需要开发高活性的催 化剂. 为此, 他们 ${ }^{[52]}$ 设计了一种原位析出的具有强金 属-载体相互作用(SMSI)的 Ru/SDC 催化剂. 在实验过程 中 $\mathrm{Ru}$ 催化剂被原位还原, $\mathrm{Ru}$ 从萤石相晶格中以金属态 析出，聚集成 3 5 $\mathrm{nm}$ 的纳米颗粒，并倾向于待在载体 的台阶面, 如图 8 所示. 为了评价 $\mathrm{Ru} / \mathrm{SDC}$ 和 Ni/SDC 催 化剂分别对水分解反应和氢氧化反应的催化活性, 他 们 ${ }^{[52]}$ 设计了四个带有不同催化剂的 SDC-SSCF 非对称 膜反应器. 结果表明对于水分解反应和氢氧化反应, $\mathrm{Ru} / \mathrm{SDC}$ 催化剂比 $\mathrm{Ni} / \mathrm{SDC}$ 的催化活性高.

\section{5 水分解反应参与的其他 OTM 反应器}

研究者们还开发了其他几种涉及水分解反应的 OTM 反应器，如图 9 所示. 合成氨和 Fischer-Tropsch 合 成制备液体燃料是两个重要的化工过程，相应的合成气 制备十分关键. 现有工业制备氨合成气 $\left(V_{\mathrm{H}_{2}} / V_{\mathrm{N}_{2}}=3\right)$ 至 少需要六步, 制备液体然料合成气 $\left(V_{\mathrm{H}_{2}} / V_{\mathrm{CO}}=2\right)$ 至少需 要三步, 工艺复杂且能耗高 (以合成氨过程为例, 制备 氨合成气的能耗占合成氨过程总能耗的 $84 \%$ ). 此外, 氨 合成气制备过程伴随大量 $\mathrm{CO}_{2}$ 的排放. 针对上述问题, $\mathrm{Li}$ 等 ${ }^{[36}$ 创新性地提出了在混合导体 OTM 反应器中一步 同时制备氨合成气和液体燃料合成气的概念，即使用 $\mathrm{H}_{2} \mathrm{O} / \mathrm{Air}$ 以及 $\mathrm{CH}_{4}$ 作为 $\mathrm{BCF}$ 膜两侧的原料气，通过调控 水和空气的比例，同时得到氨合成气及液体燃料合成气 (图 9a). 在高温下水分解产生的氧气和空气中的氧气一 起渗透到另一侧，和 $\mathrm{CH}_{4}$ 反应生成液体燃料合成气. 水 分解产生的 $\mathrm{H}_{2}$ 和空气中剩余的 $\mathrm{N}_{2}$ 经过干燥, 得到具有 
合适 $\mathrm{H}_{2} / \mathrm{N}_{2}$ 比的氨合成气. 在 $925{ }^{\circ} \mathrm{C}, \mathrm{BCF}$ 膜反应器中 可获得高达 $18.8 \mathrm{~mL} \cdot \mathrm{cm}^{-2} \cdot \mathrm{min}^{-1}$ 的氨合成气和 45.6 $\mathrm{mL} \cdot \mathrm{cm}^{-2} \cdot \mathrm{min}^{-1}$ 的液体燃料合成气生成速率. 相比于工业 制备氨合成气和液体燃料合成气过程, 该膜反应器过程 高度强化, 能耗节省高达 $63 \%$ [ ${ }^{[36]}$. 但是 BCF 材料在该类 膜反应器的应用中很难保持长期稳定性, Z Zhou ${ }^{[109]}$ 和
$\mathrm{Li}^{[110]}$ 等陆续考察了 $\mathrm{Ce}_{0.8} \mathrm{Sm}_{0.2} \mathrm{O}_{2-\delta}-\mathrm{Sr}_{2} \mathrm{Fe}_{1.5} \mathrm{Mo}_{0.5} \mathrm{O}_{5+\delta}$ 、 SDC-SSAF 双相膜在该类膜反应器中的应用, 其中 SDC-SSAF 非对称膜在 $925{ }^{\circ} \mathrm{C}$ 的两种合成气的制备速 率分别达到 20.4 和 $51.0 \mathrm{~mL} \cdot \mathrm{cm}^{-2} \cdot \mathrm{min}^{-1}$. 在以后的研究 中, 开发在强还原气氛下具有长期稳定性的膜材料仍是 该领域中的研究重点.
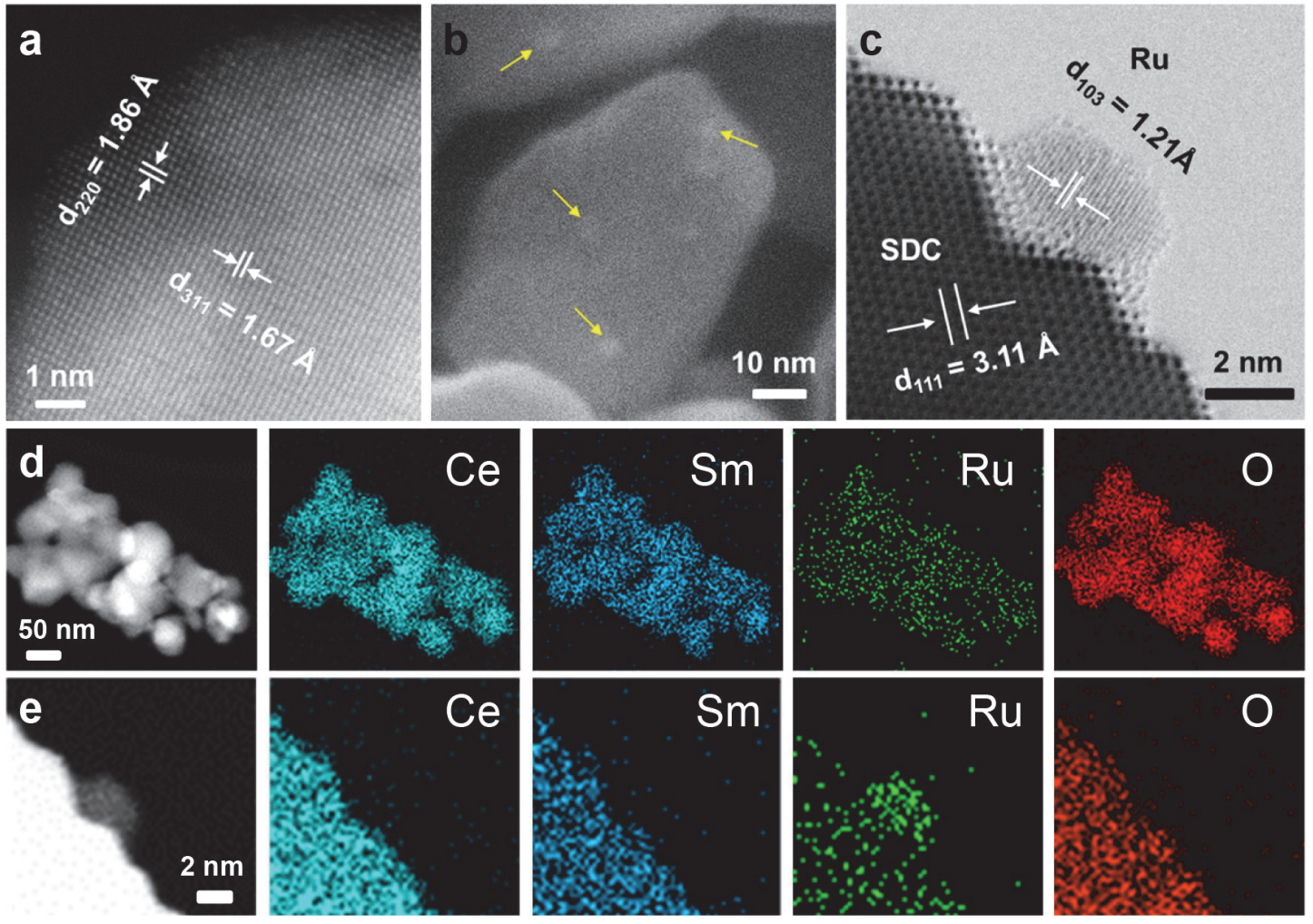

图 $8 \mathrm{Ru} / \mathrm{SDC}$ 样品的电子显微图像 ${ }^{[52]}$. (a, d) 空气气氛下制备的 $\mathrm{Ru} / \mathrm{SDC},(\mathrm{b}, \mathrm{c}$ 和 e)还原后的 Ru/SDC, (a 和 c)扫描透射电镜(STEM)图, (b)高分辨 扫描电镜(HRSEM)图, (d 和 e)能量色散 X 射线(EDX)元素成像图

Figure 8 Electron microscopy images of the Ru/SDC samples ${ }^{[52]}$. (a, d) Ru/SDC prepared in air, (b, c, e) reduced Ru/SDC, (a, c) scanning transmission electron microscopy (STEM) images, (b) high-resolution scanning electron microscopy (HRSEM) image, and (d, e) energy dispersive X-ray (EDX) element mapping images. Reprinted with permission from ref. [52], (C) 2019 Elsevier

a

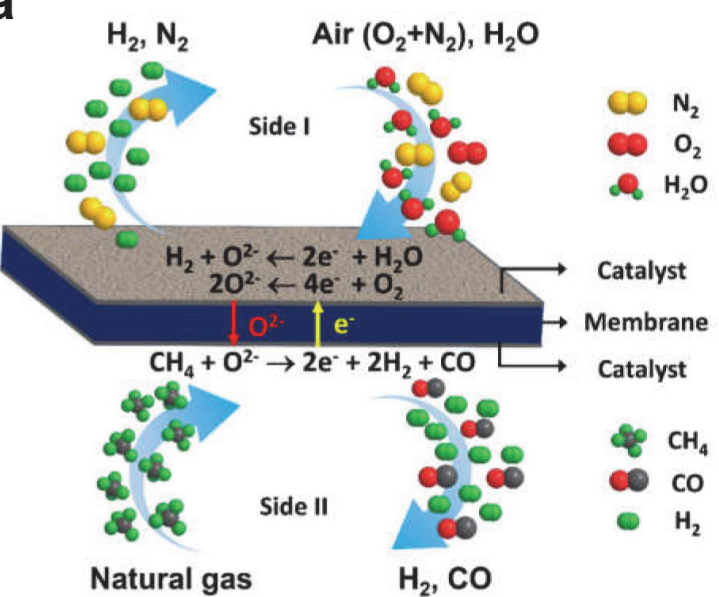

b

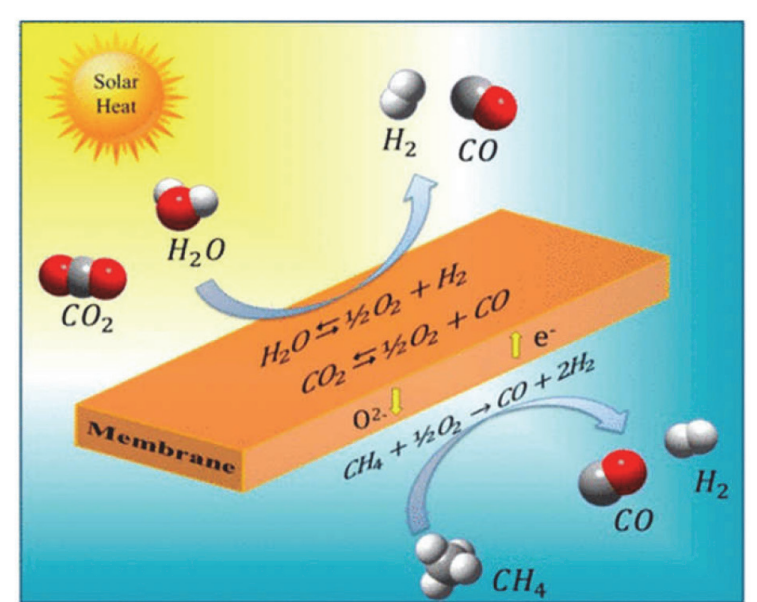

图 9 两种水参与的透氧膜反应器. (a)通过分解水和空气同时得到氨合成气 $\left(V_{\mathrm{H}_{2}} / V_{\mathrm{N}_{2}}=3\right)^{[36]}$, (b) 通过分解水和二氧化碳得到合成气 [40]

Figure 9 Two types of water-involved oxygen transport membrane reactors. (a) Synthesis gases production for ammonia $\left(V_{\mathrm{H}_{2}} / V_{\mathrm{N}_{2}}=3\right)^{[36]}$, (b) syngas production by splitting of $\mathrm{H}_{2} \mathrm{O}$ and $\mathrm{CO}_{2}{ }^{[40]}$. (a) Reprinted with permission from ref. [36], (C) 2016 John Wiley and Sons, (b) Reprinted with permission from ref. [40], (C) 2017 American Chemical Society 
Liang 等 ${ }^{[00]}$ 在 $\mathrm{Ce}_{0.9} \mathrm{Pr}_{0.1} \mathrm{O}_{2-\delta}-\mathrm{Pr}_{0.6} \mathrm{Sr}_{0.4} \mathrm{FeO}_{3-\delta}$ (CPO$\mathrm{PSFO}$ ) 双相膜反应器两侧分别通入 $\mathrm{H}_{2} \mathrm{O} / \mathrm{CO}_{2}$ 混合物和甲 烷, 两侧可同时得到合成气(图 9b). 在该体系中 $\mathrm{H}_{2} \mathrm{O}$ 和 $\mathrm{CO}_{2}$ 发生热分解反应, 分别生成 $\mathrm{H}_{2} / \mathrm{O}_{2}$ 和 $\mathrm{CO} / \mathrm{O}_{2}$, 生成的 $\mathrm{O}_{2}$ 通过 OTM 传输到另一侧和甲烷发生部分氧化反应. 根据不同需求来调控水和 $\mathrm{CO}_{2}$ 的比例, 可得到不同 $\mathrm{H}_{2} / \mathrm{CO}$ 比例的合成气. 当 $V_{\mathrm{H}_{2} \mathrm{O}} / V_{\mathrm{CO}_{2}}$ 为 5 时, $\mathrm{H}_{2} \mathrm{O} / \mathrm{CO}_{2}$ 分 解反应在 $930{ }^{\circ} \mathrm{C}$ 得到 $1.3 \mathrm{~mL} \cdot \mathrm{cm}^{-2} \cdot \mathrm{min}^{-1}$ 的合成气 $\left(V_{\mathrm{H}_{2}} / V_{\mathrm{CO}}=2\right)$.

\section{6 总结与展望}

基于氢气在能源体系中的重要地位, 氢气制备及分 离成为研究的热点. 混合导体 OTM 反应器可将水分解 反应和多种耗氧反应相耦合, 为氢气制备和分离提供了 新思路. 本文综述了近年来涉及水分解反应的混合导体 OTM 反应器的研究, 对影响水分解速率的关键因素进 行了探讨, 有助于深入认识该膜反应器过程和为提高水 分解速率指明方向.

混合导体 OTM 材料是该类膜反应器的最重要组成 部分, 发展同时具有稳定性好和透氧量高的材料是该领 域极具挑战性的课题. 本文详细总结了 OTM 材料在水 分解反应中的应用. 单相 OTM 材料的组成简单, 且透 氧量较高, 有利于移除水分解反应产生的氧. 但是多数 单相膜材料在还原气氛下的稳定性较差. 近年来开发的 双相膜材料在还原气氛下具有优异的稳定性, 但是相应 的膜反应器性能不高. 膜厚度、电导率、氧分压和温度 等是影响氧渗透过程的关键因素, 进而影响水分解性 能. 本文总结了以上因素对水分解性能的影响. 其中通 过降低膜的厚度减少体相扩散阻力可以增大氧渗透性 能, 进而增加水分解速率. 常用的方法是制备具有超薄 致密层的非对称膜来提升膜反应器的性能. 但是在该类 膜反应器中, 体相扩散阻力占比较小, 包括水分解反应 和氧移除反应在内的表面反应阻力成为限制膜反应器 性能的决速步. 非对称膜的载体层的孔隙率以及孔径大 小影响气体扩散, 造成的浓差极化进而会影响水分解性 能. 未来的研究应该采用合理策略来优化孔结构. 为了 大幅提高膜反应器的水分解速率, 在以后的研究中仍需 要设计新型的具有稳定性好和氧渗透性高的膜材料. 另 外, 膜反应器的类型也是影响水分解速率的一个重要因 素. 目前的研究中分别涉及平板膜、管状膜和中空纤维 膜, 但是使用的都是不同膜材料, 没有直接考察膜反应 器的类型对水分解制氢的影响. 由于平板膜反应器中反 应物和膜的接触时间较短, 水分解反应的转化率受限. 在管状膜或者中空纤维膜反应器中采用对流的进料方 式将会使膜反应器两端持续保持高氧分压梯度, 进而保 持高的水分解转化率. 在以后的研究中有必要考察不同 膜反应器类型对水分解速率及转化率的影响.

通过提高水分解反应和氧移除反应来减少表面反
应阻力的影响, 是提高膜反应器中水分解性能的有效手 段. 设计合成出具有高催化活性、低成本的催化剂在实 际应用中有重要意义. 目前已有多篇研究涉及对水分解 反应有较高催化活性的催化剂的开发. 对比( $\mathrm{Fe} 、 \mathrm{Co}$ 、 $\mathrm{Ni}) / \mathrm{SDC}$ 非贵金属催化剂, Ni/SDC 催化剂具有最高的催 化活性. 原位析出的 $\mathrm{Ru} / \mathrm{SDC}$ 贵金属催化剂的活性高于 非贵金属 $\mathrm{Ni} / \mathrm{SDC}$ 催化剂. 目前对催化剂的研究较少且 不够深入, 对催化反应机理的认识仍较缺乏. 在以后的 研究中, 应深入研究水分解反应的机理, 为高活性催化 剂设计提供指导.

总之, 混合导体 OTM 反应器中的水分解反应为氢 气的制备和分离提供了新方向. 正如前面总结的一样, 在膜材料、膜结构和催化剂等方面仍有很大提升空间. 在未来的研究中, 需重点优化膜材料、膜结构和催化剂 等, 获得较高的水分解速率和优异的稳定性, 为该类膜 反应器的实际应用打好坚实基础.

\section{作者简介}

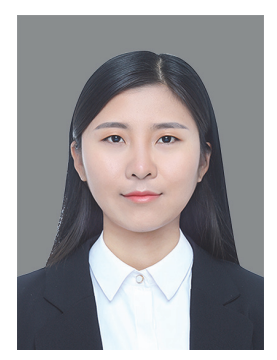

蔡莉莉, 博士, 2019 年 6 月于中国科学院大连化学物理研 究所获理学博士学位, 目前在中国科学院大连化学物理研究 所从事博士后工作, 主要研究方向为催化膜反应器.

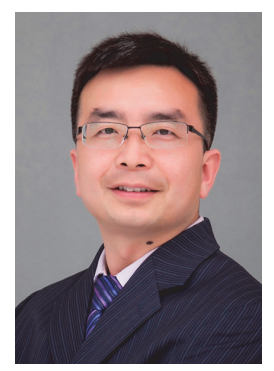

朱雪峰, 研究员, 博士生导师. 2006 年 12 月于中国科学院 大连化学物理研究所获理学博士学位. 主要从事用于气体分 离的致密陶瓷膜、膜催化及相关电催化方面的研究.

\section{References}

[1] Zhu, X. F.; Li, M. R.; Liu, H. Y.; Zhang, T. Y.; Cong, Y.; Yang, W. S. J. Membr. Sci. 2012, 394-395, 120.

[2] Geffroy, P.-M.; Fouletier, J.; Richet, N.; Chartier, T. Chem. Eng. Sci. 2013, 87, 408.

[3] Sunarso, J.; Baumann, S.; Serra, J. M.; Meulenberg, W. A.; Liu, S.; Lin, Y. S.; Diniz da Costa, J. C. J. Membr. Sci. 2008, 320, 13.

[4] Shao, Z. P.; Yang, W. S.; Cong, Y.; Dong, H.; Tong, J. H.; Xiong, G. X. J. Membr. Sci. 2000, 172, 177.

[5] Tan, X. Y.; Liu, N.; Meng, B.; Liu, S. M. J. Membr. Sci. 2011, 378, 308 . 
[6] Zeng, P. Y.; Ran, R.; Chen, Z. H.; Gu, H. X.; Shao, Z. P.; Liu, S. M. AIChE J. 2007, 53, 3116.

[7] Zhu, Y.; Cai, L. L.; Li, W. P.; Cao, Z. W.; Li, H. B.; Jiang, H. Q.; Zhu, X. F.; Yang, W. S. AIChE J. 2020, 66, e16291.

[8] Wang, S.; Shi, L.; Xie, Z. A.; Wang, H. Q.; Lan, Q.; He, Y.; Yan, D.; Zhang, X.; Luo, H. X. Chin. Sci. Bull. 2019, 64, 1651 (in Chinese). (王舒, 石否, 谢沚昂，王好奇，蓝琪，何缘，严冬，张杏，罗惠霞， 科学通报, 2019, 64, 1651.)

[9] Shi, L.; Wang, S.; Lu, T. N.; He, Y.; Yan, D.; Lan, Q.; Xie, Z. A.; Wang, H. Q.; Li, M.-R.; Caro, J.; Luo, H. X. J. Alloy. Compd. 2019, 806,500 .

[10] Luo, H. X.; Klande, T.; Cao, Z. W.; Liang, F. Y.; Wang, H. H.; Caro, J. J. Mater. Chem. A 2014, 2, 7780.

[11] Yang, Z. B.; Ding, W. Z.; Zhang, Y. Y.; Lu, X. G.; Zhang, Y. W.; Shen, P. J. Int. J. Hydrogen Energy 2010, 35, 6239.

[12] Zhang, Y. W.; Liu, J.; Ding, W. Z.; Lu, X. G. Fuel 2011, 90, 324.

[13] Zhang, G. R.; Jin, W. Q.; Xu, N. P. Energy 2018, 4, 848.

[14] Zhu, X. F.; Yang, W. S. Adv. Mater. 2019, 31, 1902547.

[15] Zhu, X. F.; Yang, W. S. AIChE J. 2008, 54, 665.

[16] Zhu, X. F.; Liu, Y.; Cong, Y.; Yang, W. S. Solid State Ionics 2013, $253,57$.

[17] Luo, H. X.; Jiang, H. Q.; Klande, T.; Cao, Z. W.; Liang, F. Y.; Wang, H. H.; Caro, J. Chem. Mater. 2012, 24, 2148.

[18] Zhu, X. F.; Cong, Y.; Yang, W. S. J. Membr. Sci. 2006, 283, 38.

[19] Zhu, X. F.; Wang, H. H.; Cong, Y.; Yang, W. S. Catal. Lett. 2006, $111,179$.

[20] He, Z. Y.; Li, C. Q.; Chen, C. S.; Tong, Y. C.; Luo, T.; Zhan, Z. L. J. Power Sources 2018, 392, 200.

[21] Cao, Z. W.; Jiang, H. Q.; Luo, H. X.; Baumann, S.; Meulenberg, W. A.; Assmann, J.; Mleczko, L.; Liu, Y.; Caro, J. Angew. Chem. Int. Ed. 2013, 52, 13794.

[22] Jiang, H. Q.; Cao, Z. W.; Schirrmeister, S.; Schiestel, T.; Caro, J. Angew. Chem. Int. Ed. 2010, 49, 5656.

[23] Wang, H. H.; Cong, Y.; Yang, W. S. Chem. Commun. 2002, 14, 1468 .

[24] Schucker, R. C.; Dimitrakopoulos, G.; Derrickson, K.; Kopeć, K. K.; Alahmadi, F.; Johnson, J. R.; Shao, L.; Ghoniem, A. F. Ind. Eng. Chem. Res. 2019, 58, 7989.

[25] Préz-Ramírez, J.; Vigeland, B. Angew. Chem. Int. Ed. 2005, 44, 1112.

[26] Wang, H. B.; Gopalan, S.; Pal, U. B. Electrochim. Acta 2011, 56, 6989.

[27] Li, W. P.; Cao, Z. W.; Cai, L. L.; Zhang, L. X.; Zhu, X. F.; Yang, W. S. Energy Environ. Sci. 2017, 10, 101.

[28] Jiang, H. Q.; Wang, H. H.; Werth, S.; Schiestel, T.; Caro, J. Angew. Chem. Int. Ed. 2008, 47, 9341.

[29] Jiang, H. Q.; Wang, H. H.; Liang, F. Y.; Werth, S.; Schirrmeister, S.; Schiestel, T.; Caro, J. Catal. Today 2010, 156, 187.

[30] Wu, X.-Y.; Cai, L. L.; Zhu, X. F.; Ghoniem, A. F.; Yang, W. S. J. Adv. Manuf. Process. 2020, 2, e10059.

[31] Wu, X.-Y.; Ghoniem, A. F.; Uddi, M. AIChE J. 2016, 62, 4427.

[32] Jia, L. J.; He, G. H.; Zhang, Y.; Caro, J.; Jiang, H. Q. Angew. Chem. Int. Ed. 2021, 60, 5204.

[33] Fang, W.; Steinbach, F.; Cao, Z. W.; Zhu, X. F.; Feldhoff, A. Angew. Chem. Int. Ed. 2016, 55, 8648.

[34] Li, W. P.; Zhu, X. F.; Cao, Z. W.; Wang, W. P.; Yang, W. S. Int. J. Hydrogen Energy 2015, 40, 3452.

[35] Liang, W. Y.; Zhou, H. Y.; Caro, J.; Jiang, H. Q. Int. J. Hydrogen Energy 2018, 43, 14478.

[36] Li, W. P.; Zhu, X. F.; Chen, S. G.; Yang, W. S. Angew. Chem. Int. Ed. 2016, 55, 8566 .

[37] Nikolaidis, P.; Poullikkas, A. Renew. Sust. Energ. Rev. 2017, 67, 597.

[38] Holladay, J. D.; Hu, J.; King, D. L.; Wang, Y. Catal. Today 2009, 139, 244.

[39] Dincer, I. Int. J. Hydrogen Energy 2012, 37, 1954

[40] Liang, W. Y.; Cao, Z. W.; He, G. H.; Caro, J.; Jiang, H. Q. ACS Sustain. Chem. Eng. 2017, 5, 8657.

[41] Thursfield, A.; Murugan, A.; Franca, R.; Metcalfe, I. S. Energy Environ. Sci. 2012, 5, 7421 .

[42] Kogan, A. Int. J. Hydrogen Energy 1997, 22, 481.

[43] Kogan, A. Int. J. Hydrogen Energy 2000, 25, 1043.

[44] Steinfeld, A. Sol. Energy 2005, 78, 603.

[45] Miller, J.; Allendorf, M.; Diver, R.; Evans, L.; Siegel, N.; Stuecker, J. J. Mater. Sci. 2008, 43, 4714.

[46] Funk, J. Int. J. Hydrogen Energy 2001, 26, 185.
[47] Naito, H.; Arashi, H. Solid State Ionics 1995, 79, 366.

[48] Song, S.-J.; Moon, J.-H.; Ryu, H.-W.; Lee, T.-H.; Dorris, S. E.; Balachandran, U. J. Ceram. Process. Res. 2008, 9, 123.

[49] Li, W. P.; Cao, Z. W.; Zhu, X. F.; Yang, W. S. AIChE J. 2017, 63, 1278 .

[50] Cai, L. L.; Hu, S. Q.; Cao, Z. W.; Li, H. B.; Zhu, X. F.; Yang, W. S. AIChE J. 2019, 65, 1088.

[51] Cai, L. L.; Zhu, Y.; Cao, Z. W.; Li, W. P.; Li, H. B.; Zhu, X. F.; Yang, W. S. J. Membr. Sci. 2020, 594, 117463.

[52] Cai, L. L.; Liu, W.; Cao, Z. W.; Li, H. B.; Cong, Y.; Zhu, X. F.; Yang, W. S. J. Membr. Sci. 2020, 599, 117702.

[53] Cai, L. L.; Wu, X.-Y.; Zhu, X. F.; Ghoniem, A. F.; Yang, W. S. AIChE J. 2020, 66, e16427.

[54] Liu, Y. T.; Tan, X. Y.; Li, K. Catal. Rev.-Sci. Eng. 2006, 48, 145.

[55] Sunarso, J.; Hashim, S. S.; Zhu, N.; Zhou, W. Prog. Energy Combust. Sci. 2017, 61, 57.

[56] Plazaola, A. A.; Labella, A. C.; Liu, Y. L.; Porras, N. B.; Tanaka, D. A. P.; Van Sint Annaland, M.; Gallucci, F. Processes 2019, 7, 128.

[57] Hashim, S. M.; Mohamed, A. R.; Bhatia, S. Adv. Colloid Interface Sci. 2010, 160, 88.

[58] Zhang, C.; Sunarso, J.; Liu, S. M. Chem. Soc. Rev. 2017, 46, 2941.

[59] Chen, C. S.; Boukamp, B. A.; Bouwmeester, H. J. M.; Cao, G. Z.; Kruidhof, H.; Winnubst, A. J. A. Solid State Ionics 1995, 76, 23.

[60] Kim, J.; Lin, Y. S. J. Membr. Sci. 2000, 167, 123.

[61] Luo, H. X.; Jiang, H. Q.; Efimov, K.; Wang, H. H.; Caro, J. AIChE J. 2011, 57, 2738.

[62] Garcia-Fayos, J.; Balaguer, M.; Serra, J. M. ChemSusChem 2015, 8, 4242.

[63] Li, W.; Liu, J. J.; Chen, C. S. J. Membr. Sci. 2009, 340, 266.

[64] Bi, X. X.; Meng, X. X.; Liu, P. Y.; Yang, N. T.; Zhu, Z. H.; Ran, R.; Liu, S. M. J. Membr. Sci. 2017, 522, 91.

[65] Cao, Z. W.; Zhu, X. F.; Li, W. P.; Xu, B.; Yang, L. N.; Zhu, X. F. Mater. Lett. 2015, 147, 88.

[66] Zhu, X. F.; Liu, H. Y.; Cong, Y.; Yang, W. S. Chem. Commun. 2012, $48,251$.

[67] Fang, W.; Gao, J. F.; Chen, C. S. Ceram. Int. 2013, 39, 7269.

[68] Wang, Z. T.; Sun, W. P.; Zhu, Z. W.; Liu, T.; Liu, W. ACS Appl. Mater. Interfaces 2013, 5, 11038.

[69] Luo, H. X.; Efimov, K.; Jiang, H. Q.; Feldhoff, A.; Wang, H. H.; Caro, J. Angew. Chem. Int. Ed. 2011, 50, 759.

[70] Xue, J.; Liao, Q.; Wei, Y. Y.; Li, Z.; Wang, H. H. J. Membr. Sci. 2013, 443, 124.

[71] Chen, T.; Zhao, H. L.; Xie, Z. X.; Xu, N. S.; Lu, Y. Ionics 2015, 21, 1683 .

[72] Chen, T.; Zhao, H. L.; Xie, Z. X.; Wang, J.; Lu, Y.; Xu, N. S. J. Power Sources 2013, 223, 289.

[73] Du, Z. H.; Ma, Y. H.; Zhao, H. L.; Li, K.; Lu, Y. J. Membr. Sci. 2019, $574,243$.

[74] Zhu, X. F.; Yang, W. S. Chin. J. Catal. 2009, 30, 801 (in Chinese). (朱雪峰, 杨维慎, 催化学报, 2009, 30, 801.)

[75] Fang, W.; Liang, F. Y.; Cao, Z. W.; Steinbach, F.; Feldhoff, A.; Angew. Chem. Int. Ed. 2015, 54, 4847.

[76] Evdou, A.; Nalbandian, L.; Zaspalis, V. T. J. Membr. Sci. 2008, 325, 704.

[77] Jiang, H. Q.; Liang, F. Y.; Czuprat, O.; Efimov, K.; Feldhoff, A.; Schirrmeister, S.; Schiestel, T.; Wang, H. H.; Caro, J. Chem 2010, 16,7898

[78] Lee, T. H.; Park, C. Y.; Dorris, S. E.; Balachandran, U. ECS Trans. 2008, 13, 379.

[79] Balachandran, U.; Lee, T. H.; Dorris, S. E. Int. J. Hydrogen Energy 2007, 32, 451.

[80] Franca, R. V.; Thursfield, A.; Metcalfe, I. S. J. Membr. Sci. 2012, 389, 173.

[81] Park, C. Y.; Azzarello, F. V.; Jacobson, A. J. J. Mater. Chem. 2006, $16,3624$.

[82] Park, C. Y.; Lee, T. H.; Dorris, S. E.; Balachandran, U. ECS Trans. 2008, 13, 393.

[83] Park, C. Y.; Lee, T. H.; Dorris, S. E.; Balachandran, U. Int. J. Hydrogen Energy 2010, 35, 4103.

[84] Park, C. Y.; Lee, T. H.; Dorris, S. E.; Balachandran, U. Int. J. Hydrogen Energy 2013, 38, 6450.

[85] Balachandran, U.; Lee, T. H.; Wang, S.; Dorris, S. E. Int. J. Hydrogen Energy 2004, 29, 291.

[86] Hong, J.; Wang, H.; Gopalan, S.; Pal, U. B. ECS Trans. 2008, 6, 1.

[87] Wang, H.; Gopalan, S.; Pal, U. B. Electrochim. Acta 2011, 56, 6989. 
[88] Patrakeev, M. V.; Bahteeva, J. A.; Mitberg, E. B.; Leonidov, I. A.; Kozhevnikov, V. L.; Poeppelmeier, K. R. J. Solid State Chem. 2003, $172,219$.

[89] Tsipis, E. V.; Patrakeev, M. V.; Kharton, V. V.; Yaremchenko, A. A.; Mather, G. C.; Shaula, A. L.; Leonidov, I. A.; Kozhevnikov, V. L.; Frade, J. R. Solid State Sci. 2005, 7, 355.

[90] Patrakeeva, M. V.; Leonidov, I. A.; Kozhevnikov, V. L.; Kharton, V. V. Solid State Sci. 2004, 6, 907.

[91] Leonidov, I. A.; Kozhevnikov, V. L.; Patrakeev, M. V.; Mitberg, E. B.; Poeppelmeier, K. R. Solid State Ionics 2001, 144, 361.

[92] Kharton, V. V.; Yaremchenko, A. A.; Shaula, A. L.; Viskup, A. P.; Marques, F. M. B.; Frade, J. R.; Naumovich, E. N.; Casanova, J. R.; Marozau, I. P. Defect Diffus. Forum 2004, 226-228, 141.

[93] Kozhevnikov, V. L.; Leonidov, I. A.; Bahteeva, J. A.; Patrakeev, M. V.; Mitberg, E. B.; Poeppelmeier, K. R. Chem. Mater. 2004, 16, 5014.

[94] Fowler, D. E.; Haag, J. M.; Boland, C.; Bierschenk, D. M.; Barnett, S. A.; Poeppelmeier, K. R. Chem. Mater. 2014, 26, 3113.

[95] Haag, J. M.; Barnett, S. A.; Richardson Jr., J. W.; Poeppelmeier, K. R. Chem. Mater. 2010, 22, 3283.

[96] Yoo, J.; Kim, S.; Choi, H.; Rhim, Y.; Lim, J.; Lee, S.; Jacobson, A. J. J. Electroceram. 2011, 26, 56.

[97] Cai, L. L.; Li, W. P.; Cao, Z. W.; Zhu, X. F.; Yang, W. S. J. Membr. Sci. 2016, 520, 607.

[98] Li, W. P.; Cao, Z. W.; Zhu, X. F.; Yang, W. S. J. Membr. Sci. 2019 , $573,370$.

[99] Midilli, A.; Ay, M.; Dincer, I.; Rosen, M. A. Renew. Sust. Energ. Rev. $\mathbf{2 0 0 5}, 9,255$.
[100] Balat, M. Int. J. Hydrogen Energy 2008, 33, 4013.

[101] Wiltowski, T.; Mondal, K.; Campen, A.; Dasgupta, D.; Konieczny, A. Int. J. Hydrogen Energy 2008, 33, 293.

[102] Schulze-Küppers, F.; Baumann, S.; Meulenberg, W. A.; Stöver, D.; Buchkremer, H.-P. J. Membr. Sci. 2013, 433, 121.

[103] Kaiser, A.; Foghmoes, S. P.; Pećanac, G.; Malzbender, J.; Chatzichristodoulou, C.; Glasscock, J. A.; Ramachandran, D.; Ni, D. W. Esposito, V.; Søgaard, M.; Hendriksen, P. V. J. Membr. Sci. 2016, $513,85$.

[104] Baumann, S.; Schulze-Küppers, F.; Roitsch, S.; Betz, M.; Zwick, M.; Pfaff, E. M.; Meulenberg, W. A.; Mayer, J.; Stöver, D. J. Membr. Sci. 2010, 359, 102.

[105] Unije, U.; Mücke, R.; Niehoff, P.; Baumann, S.; Vaßen, R.; Guillon, O. J. Membr. Sci. 2017, 524, 334.

[106] Schulze-Küppersa, F.; Unije, U. V.; Blank, H.; Balaguer, M.; Baumann, S.; Mücke, R.; Meulenberg, W. A. J. Membr. Sci. 2018, 564, 218.

[107] Cai, L. L.; Cao, Z. W.; Zhu, X. F.; Yang, W. S. Green Chem. Eng doi.org/10.1016/j.gce.2020.11.003.

[108] Zhu, N.; Dong, X. L.; Liu, Z. K.; Zhang, G. R.; Jin, W. Q.; Xu, N. P. Chem. Commun. 2012, 48, 7137.

[109] Zhou, H. Y.; Liang, W. Y.; Liang, F. Y.; Jiang, H. Q. Catal. Today 2019, 331, 2 .

[110] Li, W. P.; Cao, Z. W.; Li, H. B.; Zhu, X. F.; Yang, W. S. Int. J. Hydrogen Energy 2019, 44, 4218.

(Cheng, B.) 